\title{
DAL KĒTOS AL SĒNMURV? MUTAZIONI ICONOGRAFICHE E TRANSIZIONI SIMBOLICHE DEL KĒTOS DALL'ANTICHITÀ AL MEDIOEVO (SECOLO XIII) ${ }^{1}$
}

\section{STEFANO RICCIONI}

UDC: $75.046(495) " 03 / 14 "$

Öriginal scientific paper

Manuscript received: 28. 02. 2016.

Revised manuscript accepted: 15. 04. 2016.

DOI: 10.1484/J.HAM.5.111337

\author{
S. Riccioni \\ Università Ca' Foscari, Venezia \\ Dip. Filosofia e Beni Culturali \\ Dorsoduro 3484/D, 30123 Venezia \\ Italia
}

Using literary and iconographic sources the paper discusses the image of kētos from Antiquity to Middle Ages. The kētos, according with Greek literature, was used in the myths of both Perseus and Andromeda and Heracles and Hesione. The archaic images of the sea-monster are identifiable on Corinthian vases, on which we have only heads of leonine form. From $5^{\text {th }}$ century the classical type of kētos is distinguished from all other Greek sea-monsters by a long neck, fins (also like wings), long muzzle and corrugated upper surface (like a crocodile), and leonine forelegs. Separated from histories of Andromeda and Hesione, the ketos is represented as a mount of marine gods and, especially, Nereides. The transition from Late Antiquity to Early Christian art is well represented by Aratea and by the Book of Jonah, on which the ketos was reproduced using the classic type. It served for representing Jonah's big fish on sarcophagi and catacombs paintings, according to Midrash commentary who distinguished kētos from Leviathan. During the Middle Ages (from $11^{\text {th }}-12^{\text {th }}$ century) the image of kētos changed gradually in two directions: from classical type into a kind of a pantherl dog, sometime winged, with a sea-serpent tale (Campanian ambos); or into a simple big fish as reproduced on manuscripts and italian sculptures. The article also discusses the influence of Sassanid Sēnmurv, concluding that the kètos was essentially an elaboration of models from Antiquity.

Keywords: cetus, kētos, Jonah, Medieval bestiary, sēnmurv, simorgh, whale

Nella letteratura greca il termine kētos designa il mostro acquatico e ogni animale di grossa taglia vivente nell'acqua: balena, coccodrillo, ippopotamo, cetacei e animali ibridi ${ }^{2}$. Si veda ad esempio la sommaria descrizione che fornisce Omero nell'Iliade ${ }^{3}$ e nell'Odissea ${ }^{4}$, dove l'animale è un generico mostro marino.

Nella mitologia greca, in particolare, il kētos compare principalmente in due vicende. Nella storia di Andromeda, il kētos è l'animale mostruoso inviato da Poseidone a funestare il regno di Cefeo, per punire la superbia della moglie Cassiopea, che si era paragonata per bellezza alle Nereidi. Su sugge- rimento dell'oracolo, Andromeda, figlia di Cefeo e Cassiopea, viene offerta al mostro per liberare il paese, ma sopraggiunge Perseo che armato della testa della Gorgone sconfigge il mostro marino, libera la fanciulla, della quale si innamora, e che poi sposa dopo aver sconfitto il fidanzato Fineo5. Il mito appare in Ferecide di Atene (V secolo a. C. $)^{6}$ e trova particolare divulgazione durante il secolo V a C. con i tragici: Sofocle ${ }^{7} \mathrm{e}$ Euripide $^{8}$ raccontano questa vicenda nell'Andromeda, nuovi accenni si trovano anche in Ellianico ${ }^{9}$ e Aristofane ${ }^{10}$. Nella tradizione latina il mostro marino viene menzionato nelle Metamorfosi di Ovidio ${ }^{11}$ e nei Dialoghi marini di Luciano ${ }^{12}$.

\footnotetext{
${ }^{1}$ Questo lavoro è stato realizzato nell'ambito del progetto Croatian medieval heritage in European context: mobility of artists and transfer of forms, functions and ideas (CROMART).

${ }^{2}$ La ricerca è stata effettuata sul Thesaurus Linguae Graecae (=TLG), University of California Irvine <http://www.tlg.uci.edu>. Per la bibliografia sul Kētos, E. JOLY, s.v. Kētos, Enciclopedia dell'arte antica classica e orientale, IV, Roma, 1961, p. 347; P. W. GUNDEL, in Pauly-Wissowa (dir.), Real-Encyclopädie der Classichen Alterturmswissenschaft, XI, I, 1922, cc. 364-372; J. BOARDMAN, "Very Like a Whale" - Classical Sea Monster, in Monsters and Demons in the ancient and Medieval Worlds. Papers presented in honor of Edith Porada, ed. by A. E. FARKAS, P. O. HEPER, E. B. HARRISON, Mainz on Rhine, 1987, p. 73-84; ID., s.v. Kētos, in Lexicon Iconographicum Mythologiae Classicae (=LIMC), VIII, 1, Zürich-Düsseldorf, 1997, p. 731-736; A. BREITENBACH, J. WITTE-ORR, s.v. Kētos, in Reallexikon für Antike und Christentum. Sachwörterbuch zur Auseinandersetzung des Christentums mit der Antiken Welt, hrsg. G. SCHÖLLGEN, XX, Stuttgart, 2004 [158, 2003], p. 774-799; C. BENZONI, L. MONTEMAGNO CISERI, Dalla formica alla balena: sul ciclo illustrativo del Fisiologo ambrosiano (XI secolo), in Physis. Rivista internazionale di storia della scienza, XLII, 2, 2005, p. 251-303: 295-303; T. MANSUETO, Mostri e Nereidi: cavalcando il fantastico mare, Napoli, 2009, p. 17, 22-29; A. LATINI, La storia di Giona incisa: tipi e prototipi, in Incisioni figurate della tarda antichità, Atti del convegno di studi (Roma 22-23 marzo 2012), a cura di F. BISCONTI, M. BRACONI, Città del Vaticano, 2013, p. 201-229: 201-206, per l'etimologia del termine kètos, p. 206, con bibliografia in nota 40.

${ }^{3}$ HOMERUS, Ilias, XIII, 27, XX, 147, in TLG.

${ }_{4}^{4}$ ID., Odisseia, IV, 443, 446, 452, V, 408-421; XII, 97-98, in TLG.

${ }^{5}$ A. FERRABINO, s.v. Andromeda, in Enciclopedia Italiana, III, Roma 1950 (ed. or. 1929), p. 221-222; L. ROCCHETTI, s.v. Andromeda, in Enciclopedia dell'arte antica, I, Roma, 1958, p. 362-364; K. SCHAUENBURG, s.v. Andromeda I, in LIMC, VI, 1, Zürich-München, 1981, p. 774-775; T. MANSUETO, op. cit. (n. 1), p. 26-28, con alcune varianti e precisazioni alla storia tramandata dalla letteratura.

${ }^{6}$ PHERECYDES HIST., Fragmenta (ed. K. MULLER), 86, 4, in TLG.

7 SOPHOCLES TRAG., Fragmenta (ed. S. RADT), 52, in TLG.

${ }^{8}$ EURIPIDES TRAG., Fragmenta (ed. A. NAUCK), 145, 2, in TLG.

${ }^{9}$ HELLIANICUS HIST., Fragmenta, $3 ;$; ; 10 , in TLG.

${ }^{10}$ ARISTOPHANES COMIC., Nubes (ed. H. J. DOVER), 556, in $L G$.

" PUBLIUS OVIDIUS NASO, Metamorphoseon libri, IV, 665-803, ed. a cura di A. BARCHIESI, II, Milano, 2007, p. 111-121.

${ }^{12}$ LUCIANUS SOPH, Dialogi marini (ed. M. D. MACLEOD), 14, 1, 1; 14, 1, 14; 14, 3, 10, in TLG.
} 
L'altra storia che vede protagonista il kētos è contenuta nell'Iliade ${ }^{13}$, nella Biblioteca dello Pseudo Apollodoro (II secolo) ${ }^{14}$, nella Geografia di Strabone (I secolo a. C. - I secolo) ${ }^{15}$, nelle Storie di Diodoro Siculo (I secolo a. C. $)^{16}$ e passa nella letteratura latina con lo Pseudo Igino (II secolo) ${ }^{17}$. Re Laomedonte, il fondatore di Troia, si era rifiutato di pagare il tributo a Poseidone e Apollo per la costruzione delle mura della città. Anche in questo caso, in una variante del racconto, l'oracolo aveva indicato che il solo modo per saziare il mostro era di offrire in sacrificio Esione, la figlia del re, ma giungerà Ercole (o Tlepolemo), di ritorno dalla spedizione contro le Amazzoni, ad affrontare e sconfiggere il mostro marino identificato con il kētos ${ }^{18}$.

Con Aristotele, seguito da Eliano e Plinio, il kētos sembra aver perso il carattere mitologico; esso è descritto tra $\mathrm{i}$ vivipari (insieme al delfino, al pesce bue e alla pistrice) come un enorme pesce o un cetaceo ${ }^{19}$. La letteratura greca, però, fornisce pochissime indicazioni sulle forme della creatura indicata, per lo più, come un generico mostro marino. L'unica descrizione accurata la dobbiamo a Filostrato il Giovane (seconda metà del secolo III) che, nelle Eikones, tratteggia il kètos con occhi fluorescenti, enormi e roteanti al punto da giungere fino alla fronte coperta di aculei, muso allungato e una tripla fila di denti, lunghi e affilati, di cui alcuni sono uncinati altri rivolti all'indietro per trattenere le prede. Il collo è ritorto e la testa è enorme e mostruosa; il corpo, flessibile e sinuoso, è avvolto in spire; la coda è arcuata, brillante e colorata come la vela di una nave ${ }^{20}$.

Un'altra tradizione riporta la vicenda del kētos, ovvero i Phenomena di Arato di Soli21 ${ }^{21}$ nei quali il mostro fu posto fra le stelle come ricordo dell'impresa di Perseo che liberò Andromeda ${ }^{22}$. La traduzione dell'opera di Arato in latino, da parte di Cicerone, Ovidio, Germanico (tra il I secolo a. C. e il II secolo), da Avieno nel IV secolo e, nell'VIII secolo, da un anonimo monaco di Corbie autore dell'Aratus latinus, fu alla base dei trattati astronomici diffusi in età medievale e del loro apparato illustrativo ${ }^{23}$.

Anche il Fisiologo parla del cetus ma lo associa all'Aspidocelone, un mostro marino, descritto come una sorta di serpe-testuggine, che ha due nature. La sua bocca effonde profumi per ingannare i pesci che vi si lanciano dentro, e il suo corpo è di proporzioni talmente grandi da somigliare a un'isola. In tal modo il mostro inganna i naviganti che vi ormeggiano le navi e poi accendono un fuoco per cucinare, in quel momento, risvegliato dal calore, si inabissa trascinando i malcapitati nel fondo del mare ${ }^{24}$.

Nella letteratura latina medievale, l'etimologia di cetus, spiega Isidoro di Siviglia, nel sesto libro delle Etimologie, poi confluito nella versio BIs del Fisiologo, proviene dalle enormi dimensioni del suo corpo, in riferimento alla sua grandezza. «Si tratta, infatti, di un genere di animali giganteschi, con corpi simili a montagne, come il cetaceo che inghiottì Giona il cui stomaco era talmente grande da dare l'impressione di essere all'inferno, come dice il profeta: "Ascoltò la mia voce che usciva dal ventre dell'inferno" (Ion. 2,3$) »^{25}$. Con Rabano Mauro, l'associazione tra il cetus e la storia di Giona si consolida. Nel De rerum naturis (842-847), l'epiteto cetus è chiaramente associato all'animale mostruoso che inghiotte e rigetta il profeta, e che è ritratto con le fattezze del mostro marino della tradizione greca e romana ${ }^{26}$. Sarà questa la

${ }^{13}$ HOMERUS, Ilias, XIII, 27, XX, 147, XXI, 442-46o, in TLG.

${ }_{14}$ PSEUDO APOLLODORUS, Bibliotheca, II, 5, 9, III, 12, 3, in TLG.

${ }_{15}$ STRABO, Geographia, 13.1.32, ed. by H. L. JONES, The Geography of Strabo, VI, Cambridge-London, 1920, p. 61-63: 63.

${ }^{16}$ DIODORUS SICULUS HIST., Bibliotheca historica (ed. F. VOGEL, K. T. FISCHER), IV, 32, 1, 4; IV, 42, 2, 3; IV, 42, 5, 5; IV, 42, 6, 3; XVII, 41, 5, 5, in TLG.

${ }^{17}$ PSEUDO HYGINUS, Fabulae, 9, 1-2; 31, 4, 1; 64, 1, 2; 89, 2, 1-3; 89, 3, 3, in TLG.

${ }^{18}$ C. CAPRINO, s.v. Esione, in Enciclopedia dell'arte antica, III, Roma, 1960, p. 441-442; H. SICHTERMANN, s.v. Eracle, ivi, p. 378-390; J. BOARDMAN, s.v. Kètos op. cit. (n. 1), p. 731-736. Per una più articolata descrizione della vicenda e delle sue fonti cfr. T. MANUSETO, op. cit. (n. 1), p. 23-25.

${ }^{19}$ ARISTOTELES, Historia Animalium, 9; 23; 30, in TLG. Con la stessa accezione è menzionato in Eliano (AELIANUS, Historia Animalium, IX, 49). In Plinio si tratta di un grande capodoglio, cfr. PLINIUS SECUNDUS, Naturalis Historia, XXXVI, 6. Cfr. T. MANSUETO, op. cit. (n. 1), p. 29.

${ }^{20}$ PHILOSTRATUS IUNIOR, Imagines, 12, in TLG. Un'altra descrizione si trova in Achilles Tatius, poeta erotico del secolo IV, il quale riferisce che sott'acqua erano visibili l'ombra della sua schiena, le scaglie rigonfie, la curva del collo, la cresta spinosa e le volute della coda, indicando la lunga mascella che sembra aprirsi dalla giunzione delle spalle, cfr. ACHILLES TAT., Erotici, 3, 7, in TLG. Per entrambi, cfr. J. BOARDMAN, s.v. Këtos op. cit. (n. 1), p. 731; per Filostrato il giovane, T. MANSUETO, op. cit. (n. 1), p. 24-25.

${ }^{21}$ ARATUS ASTRON. EPIC., Phaenomena (ed. J. MARTIN), 1, 354-357; 1, 387, in TLG.

${ }^{22}$ La costellazione si colloca nell'emisfero australe ed è composta da tredici stelle: due poco luminose sull'estremità della coda, cinque dalla coda sulla curva del dorso fino al basso ventre, e sei sotto il ventre, cfr. A. SANTONI, Epitome dei Catasterismi, p. 157; si veda anche s.v. Cetus, in Certissima signa cit. http:// certissimasigna.sns.it/index.php?id=36\&type=constellation\&action=view.

${ }^{23}$ F. SAXL, Verzeichnis astrologischer und mythologischer illustrierter Handschriften des lateinischen Mittelalters in römischen Bibliotheken, Heidelberg, 19151917; ID., Verzeichnis astrologischer und mythologischer illustrierter Handschriften des lateinischen Mittelalters, II, Die Handschriften der Nationalbibliothek in Wien, Wien, 1925-1926; F. SAXL, H. MEIER, Verzeichnis astrologischer und mythologischer illustrierter Handschriften des lateinischen Mittelalters, III, Handschriften in englischen Bibliotheken / Catalogue of Astrological and Mythological Illuminated manuscripts of the latin Middle Ages, III, Manuscripts in English Libraries, London, 1953; P. MCGURK, Catalogue of Astrological and Mythological Illuminated manuscripts of the latin Middle Age, IV, Astrological Manuscripts in Italian Libraries (Other than Rome), London, 1966. Su questo argomento anche ERATOSTENE, Epitome dei catasterismi. Origine delle costellazioni e disposizione delle stelle, a cura di A. SANTONI, Pisa, 2010; F. GUIDETTI, A. SANTONI (cur.), Antiche stelle a Bisanzio. Il cod. Vat. gr. 1087. Prima giornata di studi del gruppo di ricerca sui Manoscritti Astronomici illustrati, Pisa, 2013. Per una bibliografia aggiornata si veda il progetto Manoscritti astronomici illustrati, diretto da Anna Santoni, presso la Scuola Normale Superiore di Pisa. Esso prevede anche un portale informatico consultabile in rete: Certissima signa. Manoscritti astronomici illustrati, che raccoglie informazioni sui manoscritti astronomici di età medievale e sulle tematiche di studio connesse, <http://certissimasigna.sns.it/index.php>.

${ }^{24}$ F. SBORDONE, Physiologus, Milano-Genova-Roma-Napoli, 1936, p. 64-68; Physiologus, versio Y, XXX, De ceto id est Aspidoceleon, ed. F. ZAMBON, Milano 1990, pp. 56-57.

${ }_{25}$ ISIDORO DI SIVIGLIA, Etimologia o Origini, II, XII-XX, a cura di A. VALASTRO CANALE, Torino, 2004, p. 64, 65.

${ }^{26}$ HRABANUS MAURUS, De rerum naturis, V, ed. J. P. Migne, in P.L., 111, c. 237D: Sunt enim ingentia genera belluarum, et aequalia montium corpora: qualis cetus excepit Jonam, cujus alvus tantae magnitudinis fuit, ut instar obtineret inferni, dicente propheta: Exaudivit me de ventre inferi (Ioh 2, 3). 


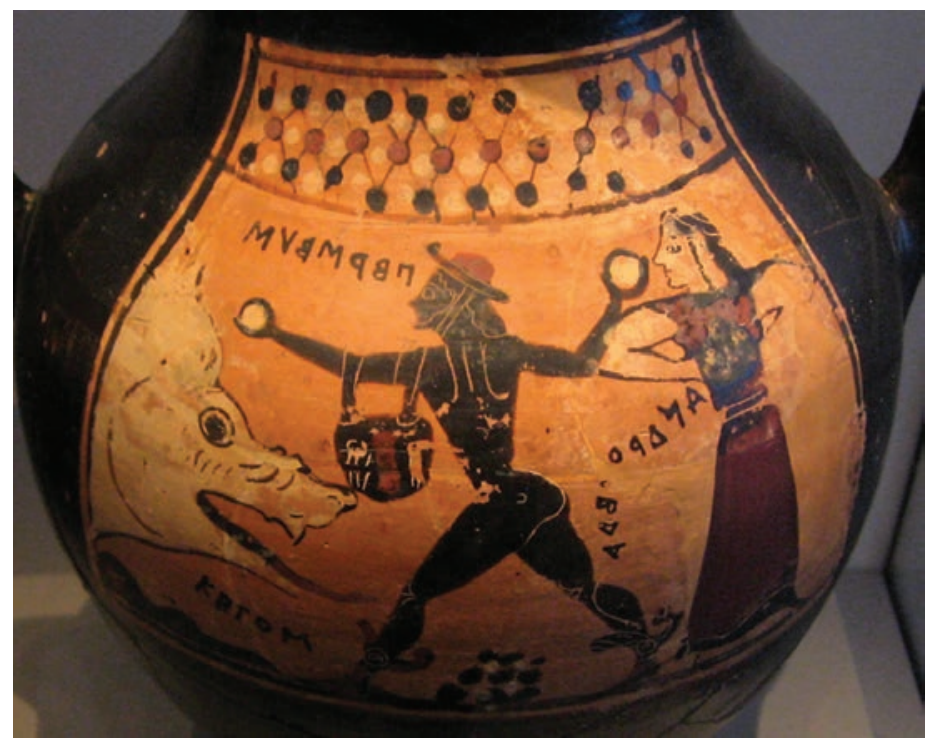

Fig. 1. Berlino, Staatliche Museen, Anfora corinzia a figure nere (da Cerveteri), Perseo e Andromeda contro il kētos, 575-550

versione tramandata durante il corso del Medioevo senza particolari alterazioni ${ }^{27}$.

\section{IL KETTOS NELLANTICHITÀ}

La raffigurazione delle prime creature marine che vengono ritenute antenate del tipo iconografico del kētos è stata identificata in un vaso proto-attico frammentario della metà del secolo VII a. C., oggi nel Museum of Fine Arts di Boston ${ }^{28}$, e in un vaso proto-corinzio proveniente da Francavilla ${ }^{29}$. In entrambi si vede solo la testa del mostro, che ha le fattezze di un cane o di un lupo. In altri esempi arcaici il muso è lungo e appuntito ${ }^{30}$, ma come accade anche per la letteratura è difficile identificare una versione univoca dell'animale ${ }^{31}$.

(Fig. 1) La prima rappresentazione certa del kētos, chiaramente identificato da un' iscrizione che ne riporta il nome, si trova in un'anfora corinzia rinvenuta a Caere (575-550 a. C.), oggi nei Musei Statali di Berlino, nella quale Andromeda accorre in aiuto di Perseo che, munito della borsa con la testa della Gorgone, scaglia sassi contro la creatura ${ }^{32}$. Anche in questo caso il mostro è raffigurato con una testa di cane/ lupo come nel contemporaneo cratere corinzio a figure nere di Boston, (fig. 2) che raffigura Ercole mentre lotta con l'animale, trafiggendolo con una raffica di frecce, per liberare Esione33. (fig. 3) In altri casi Perseo combatte con una sorta di grande amo, come in una più tarda anfora ceretana (VI-V secolo a. C.), dove il kètos inizia ad essere raffigurato con un

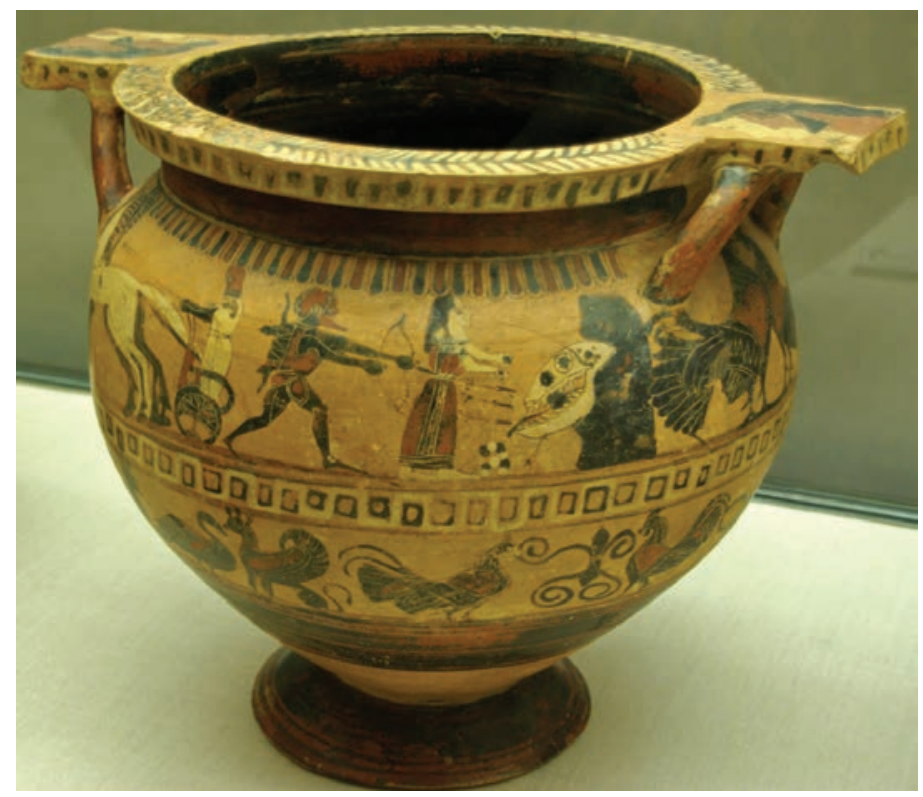

Fig. 2. Boston, Museum of Fine Arts, Cratere a figure nere, Ercole e Esione contro il kētos, 550 ca.

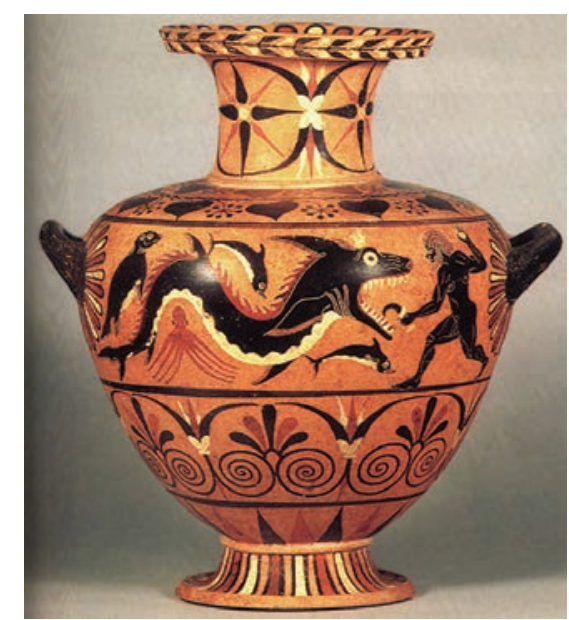

Fig. 3. Zurich, University collection, Anfora ceretana a figure nere, da Stavros S. Niarchos Collection, Perso combatte contro il kètos, VI-V secolo a. C. (da Storia Oxford dell'arte classica, a cura di J. BOARDMAN, Roma-Bari, 1995, tav. VII, fig. 71)

muso allungato e appuntito (come una sorta di coccodrillo), orecchie lunghe, un lungo corpo da pesce con le branchie e gli arti anteriori come pinne ${ }^{34}$.

(fig. 4) La serie di Andromeda legata alla roccia (un'altra iconografia la vede legata a tre pali) si trova anche in numerosi vasi italioti del secolo IV a. C., come la louthrophoros

\footnotetext{
${ }^{27}$ Per una breve antologia dell'interpretazione del termine balena/cetaceo, cfr. M. P. CICCARESE (cur.), Animali simbolici. Alle origini del bestiario cristiano, I, Bologna, 2002, p. 191-201.

${ }^{28}$ J. BOARDMAN, "Very Like a Whale" op. cit. (n. 1), p. 78, tav. XXIII, fig. 8.

${ }^{29}$ Ibid., tav. XXIII, fig. 9.

$3^{\circ}$ J. BOARDMAN, s.v. Kētos, op. cit. (n. 1), p. 735.

${ }^{31}$ K. SHEPARD, The Fish-Tailed Monster in Greek and Etruscan Art, New York, stampato privatamente, 1940, p. 28-30; E. VERMEULE, Aspects od Death in Early Greek Art and Poetry, Berkeley, 1979, p. 179-209.

$3^{2}$ Berlin, Staatliche Museen, F. 1652, cfr. J. BOARDMAN, “Very Like a Whale” op. cit. (n. 1), p. 79, tav. XXIV, fig. 10; K. Schauenburg, op. cit. (n. 4), p. 774790: 775-776; Ibid., I, 2, tav. 622, n. 1 .

33 Boston, Museum of Fine Arts, 1963.420, cfr. J. BOARDMAN, "Very Like a Whale" op. cit. (n. 1), p. 79, tav. XXIV, fig. 11. Per altri esempi anche A. Latini, op. cit. (n. 1), p. 205 n. 30.

${ }^{34}$ Zurich, University collection, Anfora ceretana a figure nere, da Stavros S. Niarchos Collection, cfr. J. BOARDMAN, "Very Like a Whale" op. cit. (n. 1), tav. XXIV, fig. 14; J. BOARDMAN, J. HUSKINSON, A. JOHNSTON et alii, Storia Oxford dell'arte classica, a cura di J. BOARDMAN, Roma-Bari, 1995 (Oxford 1993), p. 73-74, n. 71, tav. VII.
} 


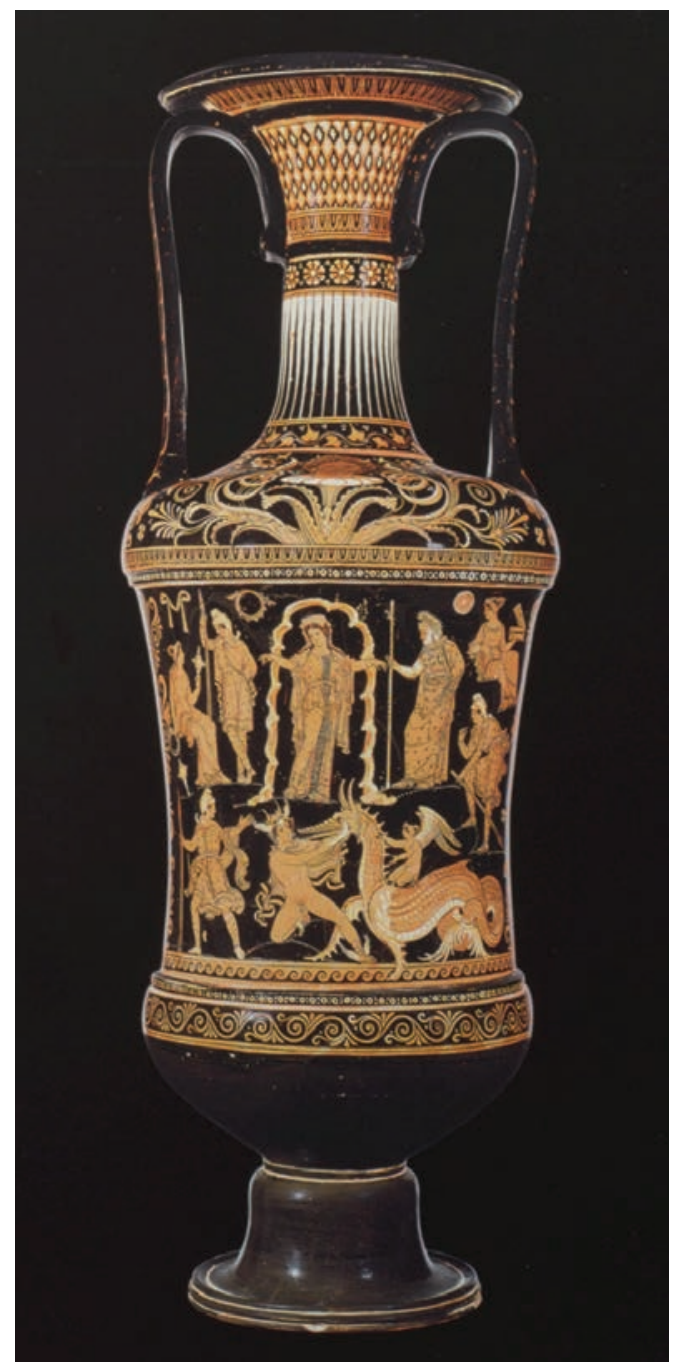

Fig. 4. Loutrophoros apula a figure rosse, Perseo e Andromeda, già Getty Museum (84.AE.996),

340-330 a.C. (da Nostoi. Capolavori ritrovati, Catalogo della mostra (Roma, Palazzo del Quirinale, 21-12/2-03, 2008), a cura di L. GODART, S. DE CARO, Roma, 2007, fig. a p. 191)

con Perseo e il Ceto proveniente dalla Puglia (340-330 a. C.) e restituita all'Italia nel 2007 dal museo Getty di Malibu (84.AE.996)35.

In questi casi, l'aspetto del kétos è quello definito "tipo classico" 36 , ovvero un animale marino con la bocca aperta (con o senza appendici) e irta di denti, muso lungo e appuntito, occhi sporgenti, orecchie puntute ed erette, pinne anteriori pronunciate (a volte anche zampe di leone), collo lungo (spesso ritorto), ventre rigonfio, corpo anguiforme, terminante con una grossa pinna, in molti casi tricuspidata.

Nelle pitture di I secolo, come a Boscotrecase ${ }^{37}$, Andromeda è raffigurata legata ad una roccia, in vesti orientali, e il kètos spunta dalle acque ma mostra solo un muso da canide,

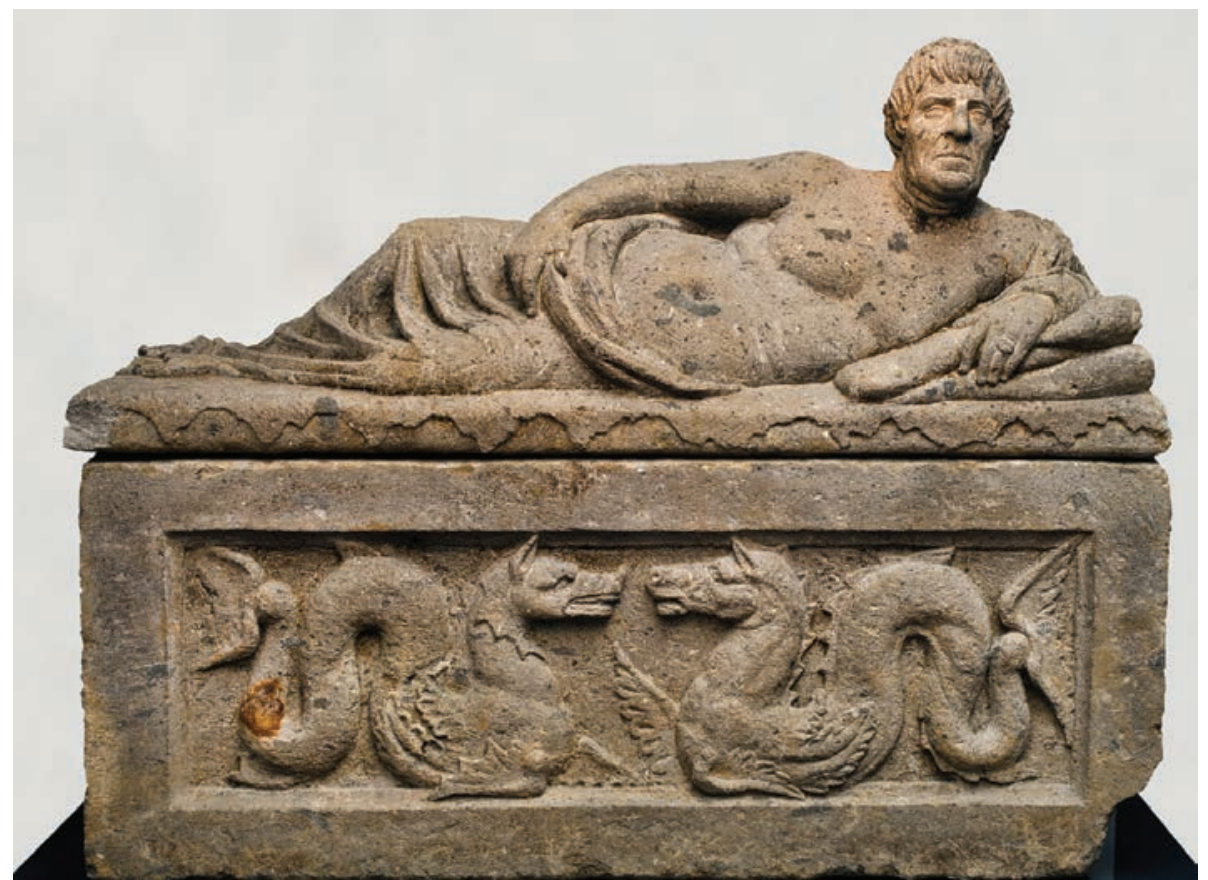

Fig. 5. Copenhagen, Ny Carlsberg Glyptothek, Sarcofago etrusco con Kētos e ippocampo, 198 a. C.

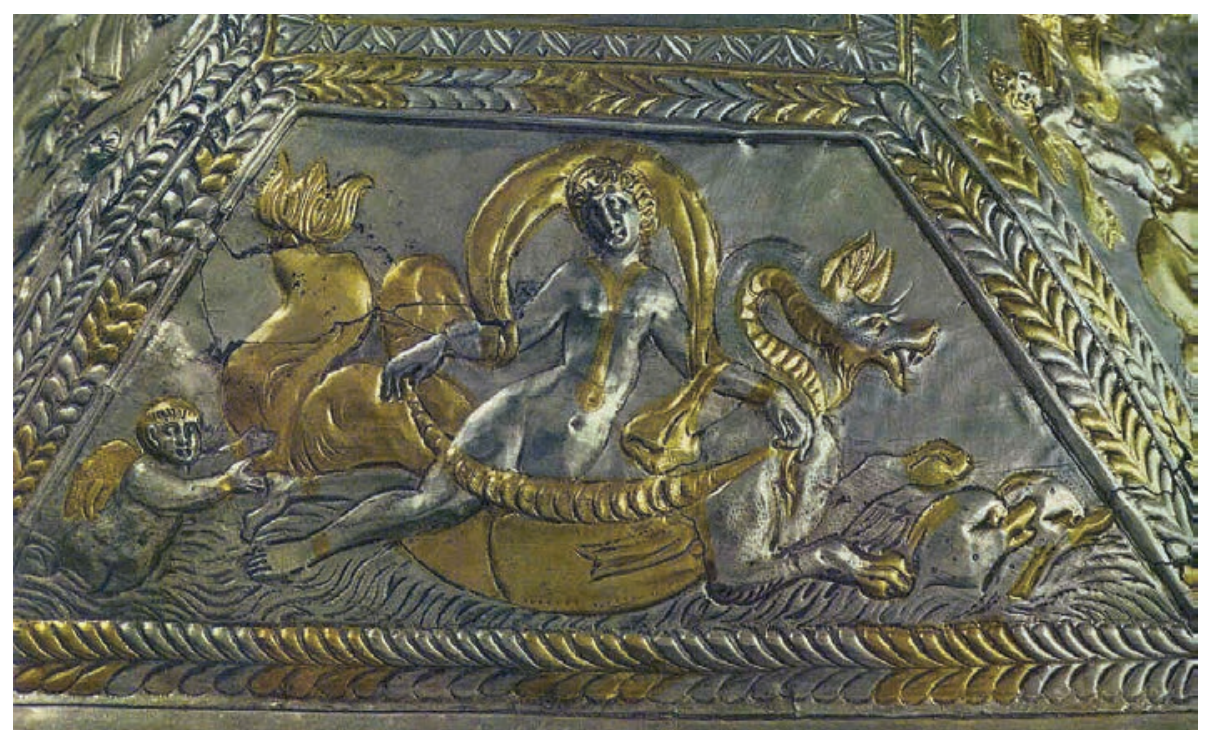

Fig. 6. Londra, British Museum, Scatola in argento di Secundus e Proiecta, Nereide su kētos, 384. (C) British Museum)

con appendici associate all'apertura buccale, come vediamo anche nella pittura murale della casa di Sacerdos Amandus di Pompei, ora al Museo Archeologico di Napoli38

Svincolato dal mito di Perseo e Andromeda il kētos è raffigurato con le fattezze "classiche", sui sarcofagi, come animale psicopompo. (fig. 5) Il suo impiego in ambito funerario è attestato fin dall'arte etrusca; il kētos occupa il lato frontale dei sarcofagi, affrontato ad altri animali in composizioni simmetriche, raffigurato con pinne lungo il corpo serpentiforme ma non avvolto in volute ${ }^{39}$.

\footnotetext{
${ }_{35}$ C. DE CARO, Scheda n. 52. Loutrophoros apula a figure rosse con Perseo e Andromeda, in Nostoi. Capolavori ritrovati, Catalogo della mostra (Roma, Palazzo del Quirinale, 21-12/2-03, 2008), a cura di L. GODART, S. DE CARO, Roma, 2007, p. 190, fig. a p. 191.

${ }^{36}$ J. BOARDMAN, s.v. Kētos op. cit. (n. 1), p. 735.

37 K. SCHAUENBURG, op. cit. (n. 4), p. 778, n. 32; Ibid., I, 2, fig. I 32.

${ }^{38}$ Ibid., p. 778 , n. 33 .

${ }^{39}$ Si vedano ad esempio i sarcofagi provenienti da Tuscania e ora al Museo Archeologico di Firenze, cfr. H. WREDE, Lebenssymbole und Bildnisse zwischen Meerwesen. Zur Entwicklung der Sepulkralsymbolik vom 5. Jahrhundert v. Chr. Bis zum 3. Jahrundert n. Chr., in H. KELLER, J. KLEINE, Festschrift für Gerhard Kleiner: zu seinem fünfundsechzigsten Geburstag am 7. Februar 1973, Tübingen, 1976, p. 147-178: 165-167; L. B. VAN DER MEER, Myths and More on Etruscan stone sarcophagi, Louvain, Dudley (MA), 2004, p. 111-112, Scheda H30, p. 129-130, Scheda H31, p. 130.
} 


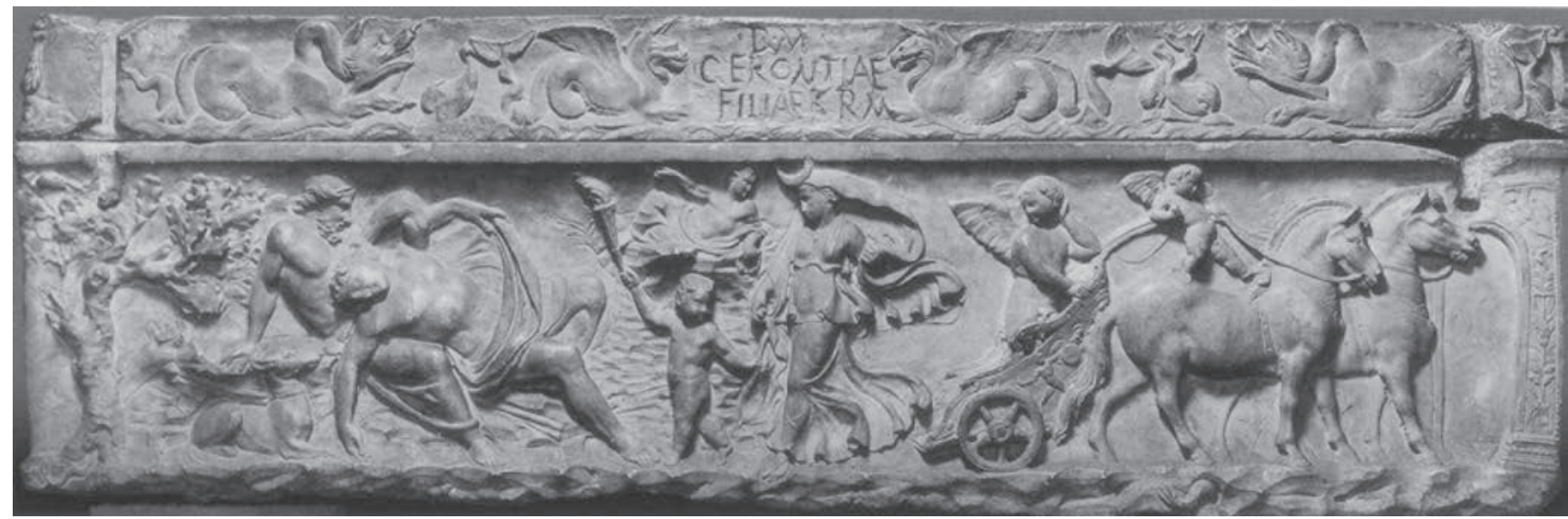

Fig. 7. Roma, Musei capitolini (inv. 325), Sarcofago, Endimione e Selene, 130-140 (da P. ZANKER, B. C. EWALD, Living with Myths. The imagery of Roman Sarcophagi, Oxford, 2012, fig. a p. 336).

(fig. 6) Spesso il kētos è cavalcato dalle Nereidi ${ }^{40}$, come nel gruppo scultoreo del Museo archeologico di Napoli (secolo I) o nei mosaici pavimentali del Museo Nazionale del Bardo a Tunisi ${ }^{41}$ (secolo II), dove viene raffigurato con un corpo anguiforme, la coda terminante con una sorta di foglia, gli arti anteriori come pinne, il muso appuntito (come una sorta di coccodrillo) che prosegue con il labbro superiore verso l'alto, e le orecchie lunghe. L'arte romana lo ritrae secondo la tradizione, con il muso che presenta un'appendice sotto la mandibola inferiore e le pinne a mo' di basette, ad esempio nel pannello dell'Ara Pacis che raffigura la Saturnia Tellus, sotto alla figura del Mare, o nel famoso cofanetto di Proiecta (IV secolo), del British Museum, dove l'animale ha zampe leonine ${ }^{42}$.

(fig. 7) Sia nelle generiche descrizioni del kètos, che nelle sue raffigurazioni, si tratta sempre di un animale marino, di norma privo di ali, sebbene si possa distinguere una variante alata del kètos, interpretando le pinne anteriori che si staccano dal corpo dell'animale e proseguono in modo pronunciato verso il basso, o in orizzontale, come delle ali ${ }^{43}$. Questa variante è frequente quando il kētos figura in veste di psicopompo sui sarcofagi ${ }^{44}$, come nella cornice superiore del sarcofago di Rapolla (seconda metà del secolo II), dove l'animale, al pari delle altre creature con le quali è allineato, ha delle pinne che si innalzano verso l'alto come delle vere e proprie ali. Esistevano, infatti, nella tradizione antica, creature dalla triplice natura: terrestre, marina e celeste, ritratte con testa di pantera/cane (ma anche di altri animali terrestri), zampe feline, coda di serpente/pesce avvolta in spire, e con ali che salgono in verticale a partire dalle zampe anteriori. Si tratta di creature anch'esse appartenenti al Thiasos marino, più genericamente indicate dalle fonti con il plurale kētē, raffigurate nell'arte greca e romana, e frequentemente collegate all'iconografia funeraria, presenti sui sarcofagi greci ed etruschi ${ }^{45}$ ma raffigurate anche in contesti non funerari, come nella "casa del tramezzo di legno" a Ercolano. ${ }^{46}$ Da questi animali, che sono il risultato della fusione di un animale terrestre o celeste con uno acquatico dal corpo anguiforme o di pesce, il kètos "classico" si distingue, per la conformazione del lungo collo e del muso ${ }^{47}$.

\section{IL KETOS NELLARTE CRISTIANA}

Con l'avvento del Cristianesimo la versione "classica" del kētos, e non il canide/pantera con coda di pesce/serpente, passa a raffigurare il "grande pesce" che inghiotte Giona..$^{4}$

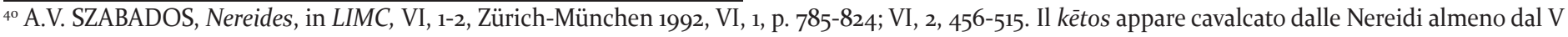
secolo a. C., cfr. Ibid., p. 790, n. 25.

${ }^{41}$ K.M.D. DUNBABIN, The Mosaics of Roman North Africa, Oxford, 1978, p. 270, tav. 13 (b), ma anche ibid., tavv. 2.3, 5.9, 50.129; J. BOARDMAN, "Very like a Whale", op. cit. (n. 1), p. 75.

${ }^{42}$ J. BOARDMAN, "Very Like a Whale" op. cit. (n. 1), p. 74.

${ }^{43}$ Si vedano gli esempi indicati da T. MANSUETO, op. cit. (n. 1), p. 51. Cfr. A.V. SZABADOS, op. cit. (n. 35), VI, 1, p. 811; VI, 2, p. 499, fig. 357; Ibid., VI, 1, p. 790; VI, 2, p. 458, fig. 40; Ibid., VI, 1, p. 797, 818; VI, 2, p. 475, fig. 164, p. 513, fig. 465.

${ }^{44}$ K. SHEPARD, op. cit. (n. 37), p. 79-84; A. RUMPF, Die Meerwesen auf den Antiken Sarkophagreliefs, Roma, 1969, p. 2, 113-116, tav. 1, fig. 1; F. CUMONT, Lux perpetua, ed. par B. ROCHETTE, A. MOTTE, Torino, 2009 (Paris, 1949), p. 333-335, per l'interpretazione di questi animali in chiave funeraria, quali traghettatori delle anime, cfr. L. B. VAN DER MEER, op. cit. (n. 36), 2004.

${ }^{45} \mathrm{Si}$ veda il sarcofago di Berlino, Staatliche Museum, inv. n. 1264, seconda metà III secolo a. C., cfr. M. BOOSEN, Etruskische Meeresmischwesen untersuchungen zu Typologie und Bedeutung, Roma, 1986, p. 29-30, n. 23, tav. VIII, n. 11, il sarcofago riporta al centro un mostro identificato come Scilla, affrontato da due pantere alate con coda fogliata. Sulla cornice superiore si notano cinque animali alati, definite ancora pantere, con testa di cane/pantera, corpo di pesce/serpente avvolto in spire e zampe anteriori. Più simile al kētos, per quanto riguarda il muso, è il drago marino raffigurato su un'urna di alabastro al Museo Archeologico di Firenze, inv. 5512, (II secolo a. C.), cfr. ibid., n. 51, p. 198, tav. XXVIII, fig. 40.

${ }^{46}$ M. COMPARETI, Ancient Iranian Decorative Textiles: New Evidence from Archaeological Investigationz and Private Collections, in The Silk Road, 13, 2015, p. 36-44: 39, fig. 7, l'autore identifica con un kētos un animale alato con testa di leone, pinne e corpo avvolto in spire.

${ }_{47} \mathrm{Si}$ veda l'esemplare di kètos che si distingue chiaramente dalle altre creature marine nel coperchio del sarcofago ai Musei Capitolini di Roma, databile agli anni 130-140 (inv. 325), cfr. P. ZANKER, B. C. EWALD, Living with Myths. The imagery of Roman Sarcophagi, Oxford, 2012 (München, 2004), p. 335-337, fig. a p. 336.

${ }^{48}$ H. LECLERCQ, s.v. Jonas, in Dictionnaire d'archéologie chrétienne et de liturgie, VII, 2, pub. par F. CABROL, H. LECLERCQ, Paris, 1927, cc. 2572-2631; A. FERRUA, Paralipomeni di Giona, in Rivista di Archeologia Cristiana, 38, 1962, p. 7-69; J. BOARDMAN, s.v. Kētos, op. cit. (n. 1), p. 736; D. MAZZOLENI, s.v. Giona, in Temi di iconografia paleocristiana, a cura di F. BISCONTI, Città del Vaticano, 2000, p. 191-193; F. BISCONTI, Nuovi paralipomeni di Giona: il
} 


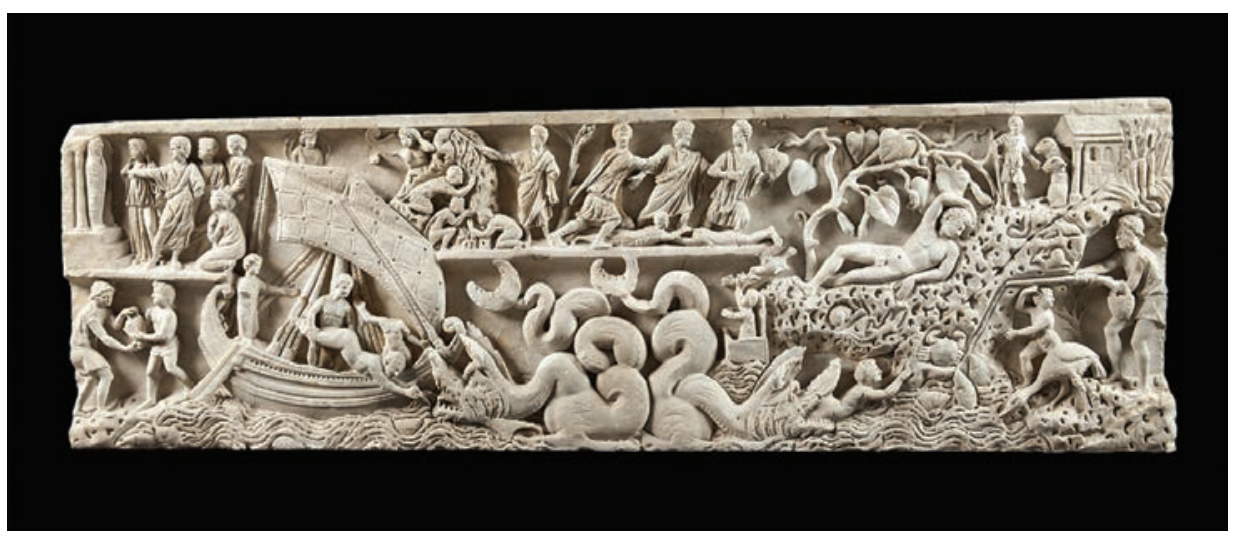

Fig. 9. Roma, Catacombe di S. Callisto, Cappelle dei Sacramenti, Cubicolo 25, Giona inghiottito e rigettato dal kètos, 200-250

(da N. BONANSEA, Simbolo e narrazione. Linee di sviluppo formali e ideologiche delliconografia di Giona tra III e IV secolo, Spoleto, 2013, tav. I, fig. 2)

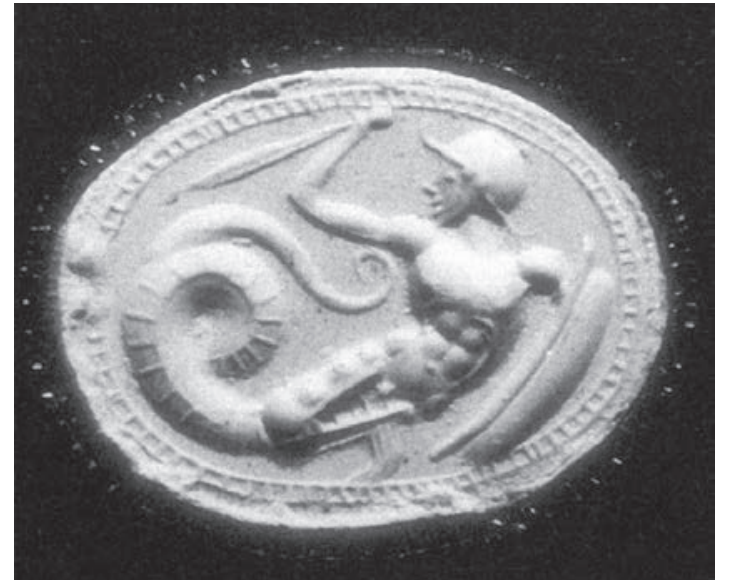

Fig. 8. Boston, Museum of Fine Arts, n. inv. 21.1203, Gemma in sardonica, Giasone rigettato dal mostro, $470-450$ a. $C$.

(da J. NEILS, Iason, in LIMC, Zürich-Berlin, 1990, VI, p. 428, fig. 33)

Gli studi sul libro di Giona hanno individuato una peculiarità nelle fonti utilizzate dall'autore; esse non si limitano a testi biblici, ma contemplano anche i miti solari che nella vicenda del profeta inghiottito e rigettato dal grande pesce esemplificano l'immersione del sole nel mare al tramonto e il suo ritorno a Oriente all'alba. Miti che accomunano la cultura egizia (la dea del cielo Nut che ingoia il sole), i miti lunari d'area mesopotamica che descrivono la scomparsa della luna per tre giorni durante la "luna nera" (discesa di Inanna agli inferi) e quella greca, con le vicende di Perseo e Andromeda, Eracle e Esione, Giasone e il drago ${ }^{49}$. Per quanto riguarda il genere letterario dell'opera, si tratta di una novella di tipo didascalico, caratterizzazione che corrisponde, nella letteratura giudaica, al midrasch ${ }^{50}$. La versione dei Settanta del libro di Giona, per indicare l'animale, usa il termine kētos mega, traduzione dell'ebraico dag gadol in latino riportato come piscis grandis ${ }^{51}$, anche se la Vulgata impiega più frequentemente il termine cetus ${ }^{52}$. In particolare Girolamo usa il termine cetus nel Vangelo di Matteo"53 la cui interpretazione contribuì al successo del libro di Giona, tenuto in gran conto dall'esegesi cristiana molto più degli altri profeti minori ${ }^{54}$. Giona infatti è l'unico tra i profeti a cui Gesù paragoni se stesso e la propria missione, per via della sua riapparizione, dopo i tre giorni nel ventre della balena, che lo ha reso un simbolo della resurrezione 55 .

La scelta di dare al dag gadol (piscis grandis) le forme del kētos mega, quello di tipo "classico", per intenderci, con il collo lungo e ritorto, la testa di coccodrillo, le zampe anteriori leonine (ma in alcuni casi anche le pinne), e il corpo avvolto in spire, con le ali/pinne, e non di un altro mostro marino, dovette essere ispirata molto probabilmente dall'interpretazione midrascica del libro di Giona ${ }^{56}$. Le fattezze della creatura, infatti, corrispondevano nella traduzione greca dei Settanta a quelle dei taninim, creature delle profondità marine variamente descritte come un serpente e un coccodrillo menzionate nella versione ebraica della Genesi ${ }^{57}$, in modo da distinguerle dal leviatano ${ }^{58}$, perché questi sarebbe stato sconfitto da Giona stesso e portato al banchetto dei

profeta e il re di Ninive in un coperchio di sarcofago del Museo cristiano di Pretestato, in Rivista di archeologia cristiana, 84, 2008 (ma 2009), p. 15-32; N. BONANSEA, Simbolo e narrazione. Linee di sviluppo formali e ideologiche dell'iconografia di Giona tra III e IV secolo, Spoleto, 2013, p. 58, ma senza particolari distinzioni tra il kētos e gli altri animali del thiasos marino; A. LATINI, op. cit. (n. 1), p. 201-229.

${ }^{49}$ A. W. WOLF, Studi sul libro di Giona, Brescia, 1982, p. 23-35; N. BONANSEA, op. cit. (n. 48), p. 4.

${ }^{50}$ A. W. WOLF, op. cit. (n. 49), p. 41-72; N. BONANSEA, op. cit. (n. 48), p. 5. Per la traduzione e il commento del testo midrashico, cfr. C. BEDINI, A. BIGARELLI (cur.), Il viaggio di Giona. Targum, Midrash, commento di Rashi, Roma, 1999.

${ }^{51}$ Ion. 2, 1: Et prceparavit Dominus piscem grandem ut deglutiret Jonam: et erat Jonas in ventre piscis tribus diebus et tribus noctibus.

${ }^{52}$ Gen. 1, 21; Job. 7, 12; Is. 27, 1; Dan. 3, 70.

${ }_{53} \mathrm{Mt}$. 12, 40: Sicut enim fuit Jonas in ventre ceti tribus diebus, et tribus noctibus, sic erit Filius hominis in corde terrce tribus diebus et tribus noctibus.

${ }^{54}$ Sull'esegesi del libro di Giona rimane fondamentale Y.-M. DUVAL, Le livre de Jonas dans la littérature chrétienne greque et latine. Sources et influences du Commentaire sur Jonas de saint Jérôme, Paris, 1973.

55 Per un aggiornato quadro dell'esegesi cristiana del libro di Giona tra i secoli III e VI, cfr. N. BONANSEA, op. cit. (n. 48), p. 13-41.

${ }^{56}$ B. NARKISS, The Sign of Jonah, in Gesta, 18 (1979), n.1, p. 63-76: 65.

${ }_{57}$ Gen. 1, 20-21. La versione dei Settanta usa il termine kētē per tradurre taninim, cfr. B. NARKISS, op. cit. (n. 56), p. 73, n. 27.

${ }^{58}$ M. M. EPSTEIN, Dreams od Subversion in Medieval Jewish Art and Literature, University Park (PA), 1997, p. 78-79; C. BEDINI, A. BIGARELLI (cur.), op. cit. (n. 5o), p. 100-101; N. SLIFKIN, Sacred Monsters: Mysterious and Mythical Creatures of Scripture, Talmud and Midrash, Brooklin (NY), 2007, p. 166-171; F. POMARICI, Mostri marini con e senza ali: considerazioni sul 'grande pesce' che ingoiò Giona, in L'officina dello sguardo. Scritti in onore di Maria Andaloro, II, Immagine, memoria, materia, a cura di G. BORDI, I. CARLETTINI, M. L. FOBELLI et alii, Roma, 2014, p. 63-68: 63-64. 
Giusti59. Tuttavia, non si può escludere che proprio il leviatano, e le descrizioni bibliche che lo indicano simile a un coccodrillo ${ }^{60}$, finiscano per condizionare anche l'iconografia del kētos che inghiotte Giona ${ }^{61}$.

Un altro elemento costituì probabilmente un modello per l'immagine di Giona inghiottito dal mostro, ovvero la raffigurazione di Giasone nelle fauci di un serpente marino ${ }^{62}$. Esempi in tal senso si ritrovano in una Kylix attica (480-470) proveniente da Cerveteri, nella quale l'eroe si trova nelle fauci del mostro al cospetto di Atena ${ }^{63} \mathrm{e}$ in una gemma in sardonica etrusca che raffigura Giasone mentre viene rigettato dal mostro (470-450 a. C.) ${ }^{64}$. (fig. 8 )

Nell'arte paleocristiana la vicenda è riportata illustrando i tre momenti salienti: quando il profeta viene gettato o cade nelle fauci della balena; quando da questa viene rigettato; quando riposa sulla spiaggia, sotto un pergolato di qiqajon - cucurbitacee (nella versione dei Settanta) - o sotto un albero (tradotto con hedera nella Vulgata) ${ }^{65}$. Queste raffigurazioni compaiono fin dagli inizi dell'arte cristiana, nel secolo III, divenendo frequenti in Occidente nel secolo IV. ${ }^{66}$ Una costante delle rappresentazioni paleocristiane è data dalla rappresentazione del "grosso pesce" o cetus che inghiotte Giona, per il quale venne sempre usato il modello iconografico del kētos "classico". ${ }^{67}$ Nell'adattamento cristiano, inoltre, l'animale che inghiotte Giona mostra spesso delle pinne che salgono dal corpo, a costituire delle ali (o in altri casi delle foglie).
La prima raffigurazione della storia di Giona che ci è giunta si trova nelle cosiddette "Cappelle dei Sacramenti" della catacomba di S. Callisto ${ }^{68}$. (fig. 9) Nei cubicoli 21, 22 e 25 , datati al 200-250, troviamo le scene in cui Giona è gettato nelle fauci del mostro marino, poi viene rigettato e infine riposa sotto la cucurbitacea ${ }^{69}$. Il kètos ha il tradizionale muso da coccodrillo, le branchie a mo' di basette, lunghe orecchie, corpo serpentiforme, ingrossato al centro, avvolto in una spira con coda tricuspidata, zampe feline e pinne.

Analoga raffigurazione si trova nei sarcofagi. Sulla fronte del sarcofago di S. Maria Antiqua (250-275) ${ }^{70}$, il kētos è raffigurato "alato", con la pinna dorsale che sale più decisamente verso l'alto, ma il corpo è sempre avvolto in spire, il muso ha la parte superiore arricciata e i denti in evidenza; in questa versione le pinne (o zampe) anteriori non sono visibili, perché coperte dalle gambe del profeta. Nell'alzata di coperchio ora nel Museo Nazionale Romano ${ }^{71}$, datato 260-290, il kētos ha il caratteristico collo ritorto, coda avvolta in una spira, pinne sul fianco e sul dorso e muso con la parte superiore arricciata. ${ }^{22} \mathrm{Nel}$ gruppo di statuette del Museum of Art di Cleveland ${ }^{73}$, esempio indicato da Boardman per illustrare il kètos anche nella variante alata ${ }^{74}$, l'animale ha due piccole pinne/ali che salgono verso l'alto ${ }^{75}$. (fig. 10) Nel sarcofago di Giona del Museo Pio Cristiano ${ }^{76}$, datato 280-300, sono rappresentati i tre momenti della storia del profeta ${ }^{77}$. Il kètos ha il capo allungato con appendici buccali, la bocca dentata, le orecchie lunghe e le branchie a mo' di basette,

\footnotetext{
${ }^{59}$ C. BEDINI, A. BIGARELLI (cur.), op. cit. (n. 50), p. 100-102; L. GINZBERG, The Legends of the Jews, 7 voll., Philadelphia, 1967-1969, IV, p. 249.

${ }^{60}$ Ez. 29, 3-5; Iob. 3, 8, 40-41.

${ }^{61}$ In alcuni casi, infatti, le fonti letterarie confondono il kētos e il leviatano e per questa via il cetus diventa immagine dell'inferno e del diavolo, cfr. S. GREGOR. MAG., Moralia sive expositio in librum job, XXIII, 39-40, ed. J. P. MIGNE, in P.L., 75, cc. 824B-825C (cetus = Inferi e maligno); RUPERTI ABBATIS TUITIENSIS, De victoria verbi Dei, XII, 14, ed. J. P. MIGNE, in P.L., 169, c. 1474A (cetus = leviatano); AUGUSTINUS HIPPONENSIS, Enarrationes In Psalmos, In Psalmum LXV Enarratio. Sermo Ad Plebem, ed. J. P. Migne, in P.L., 36, c. 791b (cetus = Inferno); cfr. INNOCENTIUS PAPA TERTIUS, Sermones de sanctis, XXIX, In solemnitate nativitatis gloriosissimae semper virginis Mariae, ed. J. P. MIGNE, in P.L., 217, c. 588B (cetus = diavolo/Satana).

${ }^{62}$ J. NEILS, Iason, in LIMC, Zürich-Berlin, 1990, V, 1 p. 632, V, 2, p. 427, fig. 30, p. 428, fig. 32-35; T. MANSUETO, op. cit. (n. 1), p. 66-68.

${ }_{3}$ Città del Vaticano, Museo Gregoriano, n. inv. 16545, cfr. Ibid., V, 2, p. 428, fig. 32.

${ }^{64}$ Boston, Museum of Fine Arts, n. inv. 21.1203, cfr. Ibid., VI, p. 428, fig. 33.

${ }^{65}$ Sulle varie traduzioni dell'arbusto biblico, cfr. G. RINALDI, I tre quadri di Giona dell'aula teodoriana, in Mosaici in Aquileia e nell'Alto Adriatico. Atti della V settimana di studi aquileiesi (Aquileia, 25 aprile - 1 maggio 1974), Udine, 1975, p. 109-130: 110-113; E. SCIROCCO, Jonah, the Whale and the Ambo. Image and Liturgy in the Medieval Campania, in The Antique Memory and the Middle Ages, ed. by I. FOLETTI, Z. FRANTOVÂ, Roma, 2015, p. 90, nota 9, con bibliografia. ${ }^{66}$ Più rare sono le raffigurazioni di Giona in cammino verso Ninive o nell'atto di salire in barca, cfr. N. BONANSEA, op. cit. (n. 48), p. 43-115.

${ }^{67}$ La ricerca sulle testimonianze dipinte si è svolta sui seguenti repertori, J. WILPERT, Le pitture delle catacombe romane, Roma, 1903; A. NESTORI, Repertorio topografico delle pitture delle catacombe romane, Città del Vaticano, 1993; V. FIOCCHI NICOLAI, F. BISCONTI, D. MAZZOLENI, Le catacombe cristiane a Roma, Regensburg, 1998.

${ }^{68} \mathrm{Ibid}$., p. 43. La ricerca sulla scultura è stata effettuata nell'ambito dei sarcofagi di produzione romana, cfr. J. WILPERT, I sarcofagi cristiani antichi, Roma, 1932; F. W. DEICHMANN, G. BOVINI, H. BRANDENBURG, Repertorium der christlich-antiken Sarkophage, I, Rom und Ostia, Wiesbaden, 1967; J. DRESKEN-WEILAND, G. BOVINI, H. BRANDENBURG, Repertorium der christlich-antiken Sarkophage, II, Italien mit einem Nachtrag. Rom und Ostia, Dalmatien, Museen der Welt, Mainz am Rhein, 1998. Per altri contesti ci siamo avvalsi di N. BONANSEA, op. cit. (n. 48).

${ }^{69}$ A. NESTORI, op. cit. (n. 62), n. 21-23, p. 106, n. 25, p. 107; N. BONANSEA, op. cit. (n. 48), scheda A4, p. 170-171, scheda A5, p. 171, tav. I, fig. 1, scheda A6, p. 171, scheda $A 8$, p. 171, tav. I, fig. 2.

${ }^{70} \mathrm{~J}$. WILPERT, I sarcofagi cristiani op. cit. (n. 63), p. 7, 20, 17, 21, tav. I, 2; III, 1; F. W. DEICHMANN, G. BOVINI, H. BRANDENBURG, op. cit. (n. 63), tav. 117 , n. 747; N. BONANSEA, op. cit. (n. 48), scheda S1, p. 191.

${ }^{71} \mathrm{~N}$. inventario 106174 (b).

${ }^{72}$ Il sarcofago proviene da Roma, Monteverde (Catacomba di Ponziano?), cfr. F. W. DEICHMANN, G. BOVINI, H. BRANDENBURG, op. cit. (n. 63), tav. 127, n. 794a-b; N. BONANSEA, op. cit. (n. 48), scheda $S_{4}$, p. 992, tav. III, Fig. 6.

73 W. D. WIXOM, Early Christian sculptures at Cleveland, in The Bulletin of the Cleveland Museum of Art, 1, 1967, p. 67-89: 77-88, l'autore propende per l'autenticità del gruppo che data al terzo quarto del secolo III; E. KITZINGER, op. cit. (n. 40), p. 657-662, l'autore riporta i dubbi sull'autenticità del gruppo che tuttavia ritiene originale, datandolo al 270-280.

${ }^{74}$ Ibid., p. 71, tav. XXI, fig. 1. In realtà Boardman definisce caratteristica "unconventional" e "inappropriate" la variante con le ali al posto delle pinne.

${ }_{75} \mathrm{Ma}$ solo per l'animale che rigetta Giona, quello che lo inghiotte invece ha pinne lungo il corpo, cfr. E. KITZINGER, The Cleveland Marbles, in I monumenti cristiani precostantiniani. Atti del IX congresso internazionale di Archeologia Cristiana (Roma 21-27 settembre 1975), Città del Vaticano, 1978, p. 653-675: 657, fig. 7

${ }^{76}$ N. inv. 31448 (ex 119).

77 J. WILPERT, I sarcofagi cristiani op. cit. (n. 63), p. 17, 26, 32, 109, 140, tav. IX, 3; F. W. DEICHMANN, G. BOVINI, H. BRANDENBURG, op. cit. (n. 63), tav. 11, n. 35; N. BONANSEA, op. cit. (n. 48), scheda $S_{32}$, p. 197, tav. IV, fig. 7.
} 


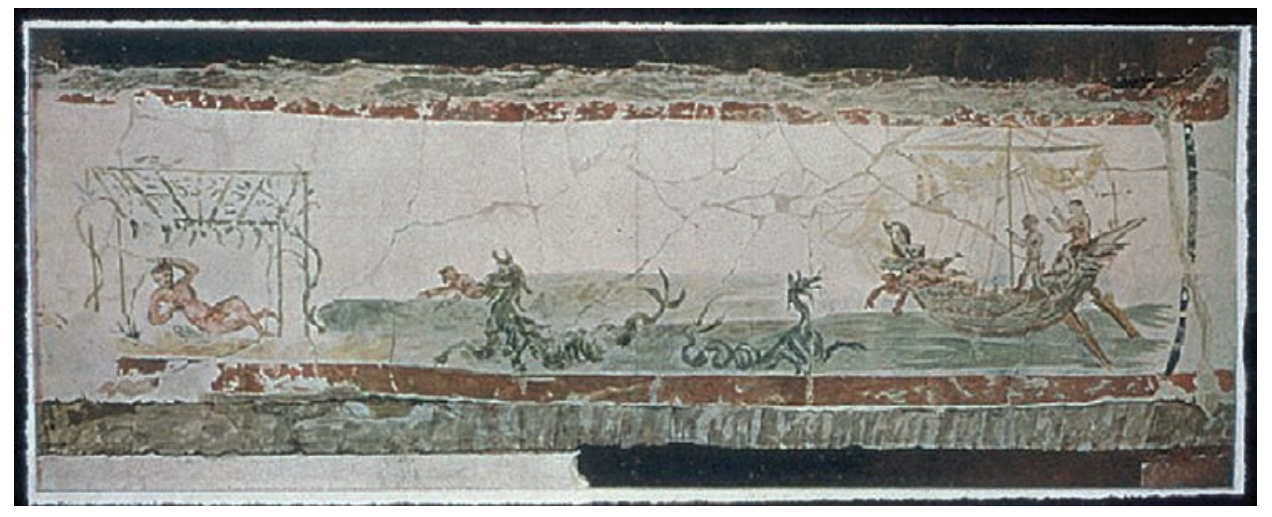

Fig. 10. Roma, Museo Pio Cristiano, n. inv. 31448 (ex 119), Sarcofago di Giona, fronte, 280-300 (da N. BONANSEA, Simbolo e narrazione. Linee di sviluppo formali e ideologiche dell'iconografia di Giona tra III e IV secolo, Spoleto, 2013, tav. IV, fig. 7)

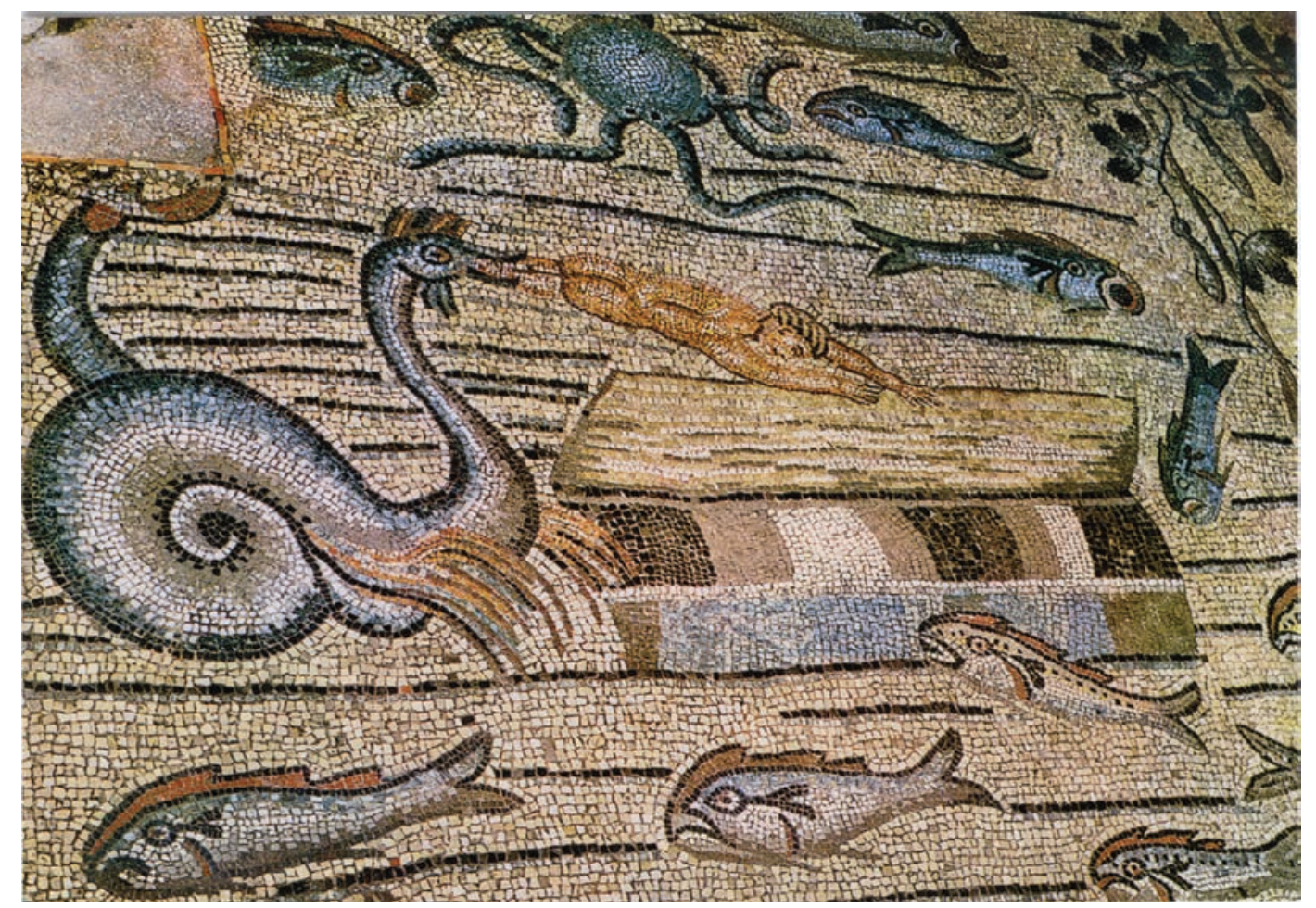

Fig. 11. Aquileia, Basilica Teodoriana, Aula Nord, Mosaico pavimentale, Giona inghiottito dal kètos, $319 \mathrm{ca}$.

zampe anteriori e pinne lungo il corpo avvolto in spire e terminante con una pinna a mezzaluna. Si tratta di un'iconografia che è rappresentata con continuità sui lati frontali dei sarcofagi, soprattutto sui loro coperchi, che contengono la storia di Giona ${ }^{78}$. Analoghe considerazioni possono farsi per la raffigurazione dell'animale sulle lastre incise ${ }^{79}$.

In esempi più tardi, come nelle pitture delle catacombe dei Santi Pietro e Marcellino, cubicolo $16^{80}$ (prima metà secolo IV), il kētos ha un muso da coccodrillo, lunghe orecchie, corpo da serpente avvolto in tre spire, con pinna terminale tricuspidata e zampe. (fig. 11) Una simile iconografia si trova nei mosaici di Aquileia (secolo IV), dove il kétos è ritratto mentre inghiotte e rigetta Giona ${ }^{81}$. In questo caso le pinne anteriori sono molto pronunciate, al punto da potersi scambiare per delle ali, ma il corpo è sempre quello del kètos classico, con muso allungato, il collo ritorto e il corpo avvolto in una spira.

\section{IL KETOS NEL MEDIOEVO}

Nella tradizione manoscritta degli Aratea e dei trattati astronomici di età medievale il kētos è raffigurato, almeno

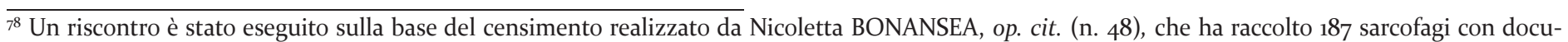
mentazione fotografica, incrociato con le immagini riprodotte in J. WILPERT, I sarcofagi cristiani op. cit. (n. 63), e F. W. DEICHMANN, G. BOVINI, H. BRANDENBURG, op. cit. (n. 63).

${ }^{79}$ A. LATINI, op. cit. (n. 1), passim.

${ }^{80}$ J. WILPERT, Le pitture op. cit. (n. 62), tav. LXV, 1, LXVIII, 1, LXIX, 1; A. NESTORI, op. cit. (n. 62), p. 51, n. 16; N. BONANSEA, op. cit. (n. 48 ), scheda A 41, p. 179-18o.

${ }^{81}$ G. RINALDI, op. cit. (n. 6o); A. QUACQUARELLI, Note esegetiche sul pavimento musivo della basilica teodoriana di Aquileia, in Aquileia nel IV secolo. Atti della XII Settimana di Studi Aquileiesi (Aquileia, 30 aprile - 5 maggio 1981), II, Udine, 1982, p. 429-462.
} 


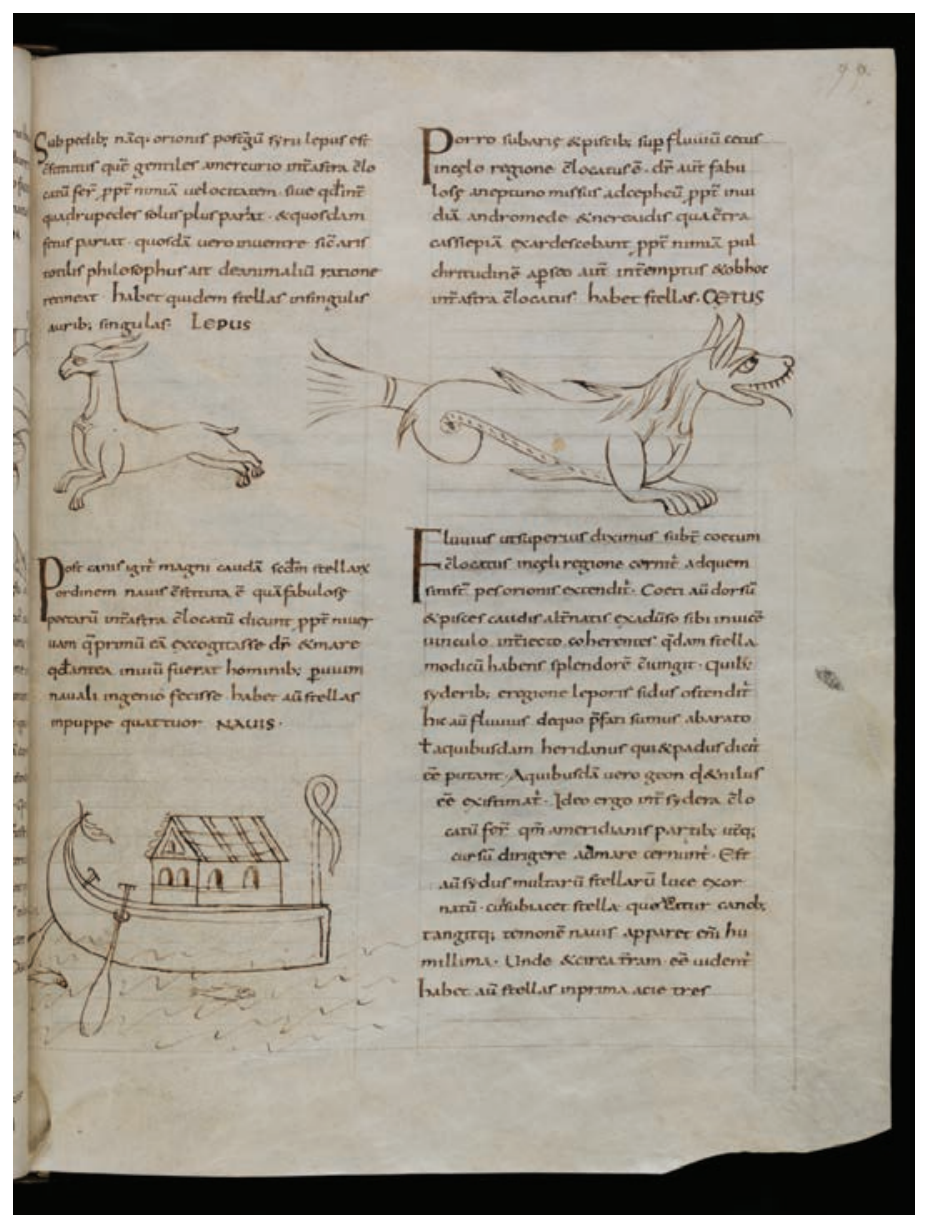

Fig. 12. St. Gallen, Stiftsbibliothek, Cod. Sang. 902, p. 95, Manoscritto miscellaneo con l'Arato latino, Cetus, metà secolo IX (www.e-codices.unifr.ch)

fino alla metà del secolo IX, ancora secondo l'iconografia del "tipo classico": il capo è allungato come quello di un coccodrillo, gli arti anteriori sono spesso delle pinne molto pronunciate ${ }^{82}$, sebbene non manchino esempi in cui l'animale è raffigurato con delle vere e proprie zampe ${ }^{83}$. Nel mano-

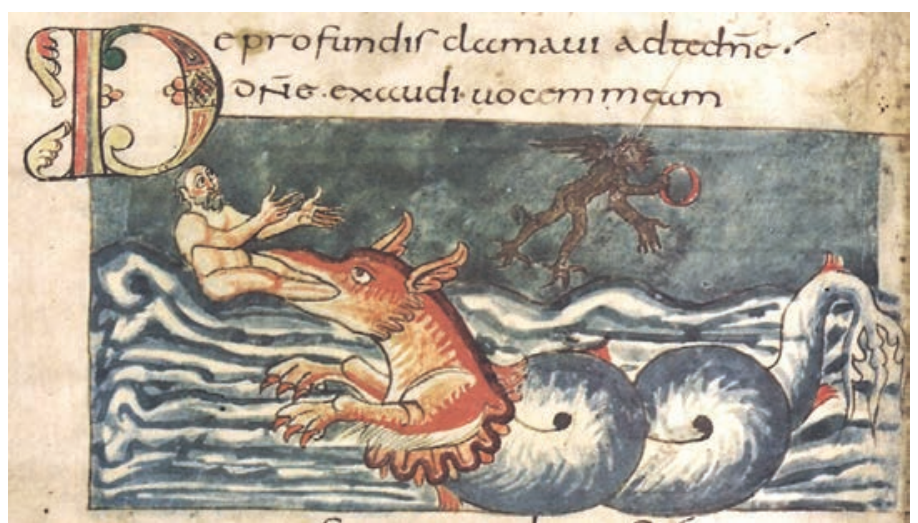

Fig. 13. Stuttgart Württemberische Landesbibliothek, Cod. bibl. fol. 23, fol. $147 v$, Giona rigettato dal kètos, $830 \mathrm{ca}$.

(http://warburg.sas.ac.uk/vpc/VPC_search/record.php?record=32230)

scritto 83 II di Colonia, del principio del secolo IX, le forme della creatura sono vicine a quelle tramandate dall'antichità: muso allungato, orecchie pronunciate, basette, pinne, e corpo avvolto in tre spire ${ }^{84}$. Segue lo stesso modello il kétos raffigurato nel manoscritto Harley 647, realizzato a Reims attorno alla metà del secolo IX e oggi conservato presso la British Library ${ }^{85}$ : la creatura è ritratta con un muso allungato, le orecchie pronunciate, le zampe unghiate e il corpo attorcigliato in una spira ${ }^{86}$.

Durante il corso del Medioevo, tuttavia, il mostro marino subisce alcune mutazioni. (fig. 12) Il codice astronomico 902, contenuto in una miscellanea realizzata per la scuola abbaziale di S. Gallo (metà del secolo IX), mostra il cetus (indicato chiaramente nel testo) con il capo di canide, un corpo anguiforme e due vere e proprie zampe anteriori ${ }^{87}$. A partire dal secolo X, e durante i secoli XI e XII, il muso si accorcia e assume le sembianze canine o leonine, mentre le pinne vengono sostanzialmente sostitute dalle zampe da cane o leone ${ }^{88}$. In esemplari più tardi, il cetus perde le caratteristiche del mostro marino di derivazione classica e viene raffigurato come un pesce ${ }^{89}$.

\footnotetext{
$\overline{8_{2}}$ Per questa ricerca mi sono avvalso del Database iconografico del Warburg Institute, <http://warburg.sas.ac.uk> dove si possono vedere le illustrazioni del cetus nei seguenti manoscritti: Cologne, Dombibliothek, 83 II, fol. 165v (798-805); Laon, Bibliothèque municipale, 422, fol. 30r, PSEUDO BEDA, De signa caeli, (inizio secolo IX); Dresden, Sächsische Landesbibliothek, Dc 183, Aratus latinus, fol. 24r, (seconda metà IX sec); Madrid, Biblioteca National de España, 3307, De ordine ac positione stellarum in signis, fol. 61r (820 ca.).

La ricerca si è svolta anche sul repertorio presente nel sito del progetto Certissima signa: http://certissimasigna.sns.it/index.php?id=36\&type=constell ation\&action=view_. Il kētos classico appare ancora nel secolo XII, cfr. F. SAXL, Verzeichnis astrologischer und mythologischer illustrierter Handschriften des lateinischen Mittelalters, II, Die Handschriften der Nationalbibliothek in Wien, Wien, 1926, p. 159-163, Cod. Vindobonensis 1260o, f. 25v, Costellazioni, tav. II, fig. 2.

${ }^{83}$ Leiden, Universiteitsbibliotheek, Voss. lat. Q. 79, GERMANICUS CAESAR, Aratea, fol. 66v (816 ca.); St. Gallen, Stiftsbibliothek, Cod. Sang. 902, f. 95 (seconda metà secolo IX).

${ }^{84}$ Köln, Erzbischöfliche Diözesan und Dombibliothek, Cod. 83-II, f.146r-171v, testo Aratea, immagine kētos, f. 165v. http://cdm.csbsju.edu/cdm/ref/ collection/HMMLClrMicr/id/176o7/rec/18.

${ }^{85}$ F. GUIDETTI, A. IAFRATE, s.v. London, British Library, ms. Harley ms 647, in Certissima Signa <http://certissimasigna.sns.it/index.php?id=6\&type $=$ manuscript\&action=view $>$.

${ }^{86}$ London, British Library, ms. Harley ms 647, f. 1or, http://www.bl.uk/manuscripts/Viewer.aspx?ref=harley_ms_647_fo1or.

${ }^{87}$ St. Gallen, Stiftsbibliothek, Cod. Sang. 902, p. 95, Manoscritto miscellaneo con l'Arato latino. Per la bibliografia, le immagini e una scheda completa, cfr. http://www.e-codices.unifr.ch/it/list/one/csg/ogoz.

${ }^{88}$ London, British Library, Harley 2506, CICERO, Aratea, fol. 42 r (994 ca.); Paris, Bibliothèque nationale de France, lat. 8663, Aratea, De ordine ac positione stellarum in signis, fol. $23 \mathrm{r}$ (inizio secolo XI); Firenze, Biblioteca Medicea Laurenziana, Plut. 29. 30, Hyginus, De Astronomia, fol. 22r (secolo XII); Vienna, Österreichische Nationalbibliothek, Cod. 51, fol. 154r, Hyginus, De Astronomia (ante 1150); F. SAXL, Verzeichnis astrologischer und mythologischer illustrierter Handschriften des lateinischen Mittelalters in römischen bibliotheken, Heidelberg, 1915, p. 70-71, Vat. lat. 643, Andromeda, tav. VII, fig. 15 (secolo XII); p. 45-59, Regin. lat. 123, f. 192v, Andromeda, tav. VII, f. 192v (secolo XII) (in questi due casi il kētos è raffigurato come un serpente); p. 59-66, Regin. lat. 309, f. 98r, Coetus, tav. XXI, fig. 44 (secolo IX).

${ }^{89}$ F. SAXL, op. cit. (n. 74), p. 132-141, Wien, Cod. 5328, f. 26v e 27 r, Andromeda, tav. VIII, fig. 15 (secolo XV); F. SAXL, H. MEIER, Verzeichnis astrologischer und mythologischer illustrierter Handschriften des lateinischen Mittelalters, III, Handschriften in englischen Bibliotheken / Catalogue of Astrological and Mythological Illuminated manuscripts of the latin Middle Ages, III, Manuscripts in English Libraries, London, 1953, tav. LXXI, fig. 177, Oxford, Rawlinson,
} 


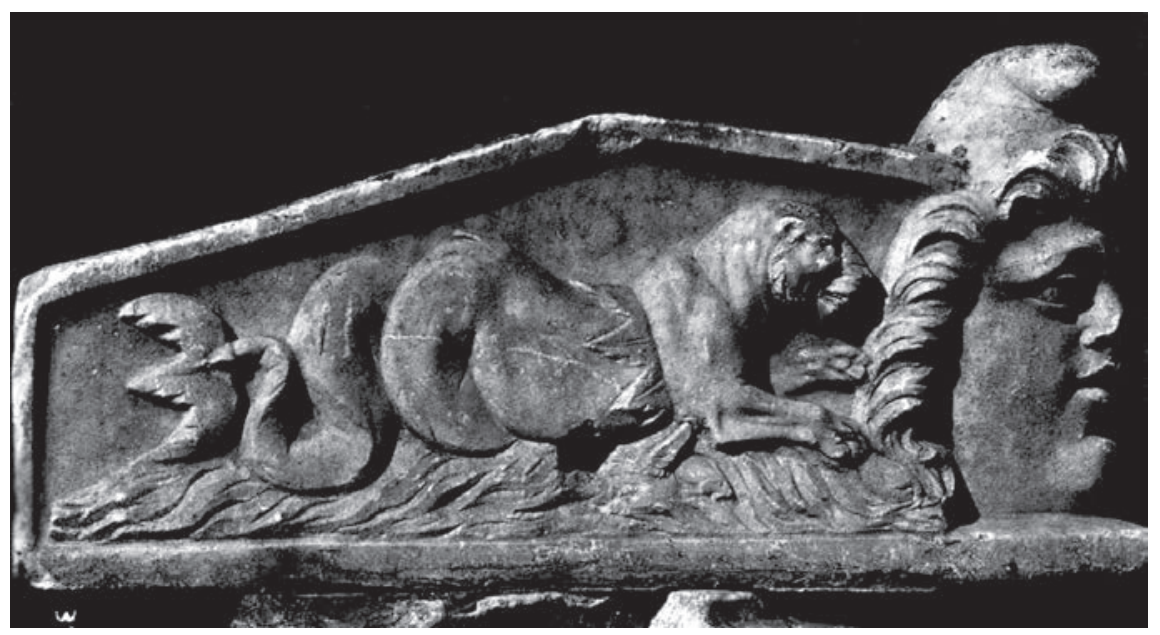

Fig. 14. Città del Vaticano, Giardino della Pigna, Frammento di sarcofago, pseudo-Kètos (da D. GLASS, Jonah in Campania. A late antique revival, in Commentari. Rivista di critica e storia dell'arte, a. XXVII, 23 1976, p. 187, fig. 10)

Tornando alla storia di Giona, il Salterio di Stoccarda, proveniente da Saint-Germain-des-Prés (830 ca.), illustra i commenti al Salmo 68, 3, quando il mostro inghiotte il profeta $^{90}$, e al Salmo 129, 1, (fig. 13) quando lo rigetta91. Il kètos classico, in quest'ultimo esempio appare adattato dalla fantasia medievale; le pinne sul dorso dell'animale che rigetta Giona, infatti, sono divenute una sorta di collarino ondulato, il corpo serpentiforme è avvolto in due spire, la pinna terminale, tricuspidata, è quasi una foglia, mentre il capo, pur restando allungato, ha perduto le caratteristiche del coccodrillo, ma mantiene le lunghe orecchie della tradizione antica. Nella copia manoscritta del De rerum naturis di Rabano Mauro, conservata a Montecassino e datata al secolo IX, invece, l'animale che inghiotte e rigetta Gione è una sorta di serpente marino con testa di canide ${ }^{92}$. Del kētos dell'antichità, in questi esempi alto medievali resta il corpo serpentiforme, ma il capo abbandona le forme classiche del coccodrillo, facendo somigliare l'animale ad un cane o a una pantera.

Queste mutazioni, in particolare quelle intervenute nell'alto medioevo, nell'ambito della produzione manoscritta, si potrebbero giustificare con la contaminazione di quei mostri marini con una doppia natura, che popolano la mitologia greca, etrusca e romana e sono frequenti nei sarcofagi, anche in quelli paleocristiani93. (fig. 14) In particolare, lo pseudo-kētos con testa da pantera/canide è spesso raffigurato da solo, con una specifica stilizzazione delle pinne che diventano foglie (oppure ali) sul dorso del corpo anguiforme.

Durante il secolo VIII, abbiamo almeno un chiaro esempio di recupero di questo animale, nella versione alata. Nel pluteo detto di Teodote, proveniente dall'oratorio di San Michele alla Pusterla94, le creature affrontate, con corpo avvolto in spire, testa di canide/pantera, zampe leonine eali, sono un chiaro recupero dei modelli largamente diffusi nell'arte funeraria antica e paleocristiana, e non necessariamente una contaminazione dell'arte orientale ${ }^{95}$.

Per quanto riguarda la storia di Giona, durante il corso del Medioevo, alcuni manoscritti di produzione greca datati al IX secolo, riportano il mostro marino ancora nelle sembianze del kétos classico ${ }^{96}$, tradizione che prosegue almeno fino al secolo XII, anche nella sua versione alata ${ }^{97}$. Tuttavia già a partire dal secolo XI, nei manoscritti latini, si afferma la tendenza a sostituire la creatura che inghiotte e rigetta il profeta con un pesce, come nella Bibbia di Roda ${ }^{98}$ e nella Bibbia Lat. 1652899 conservate nella Biblioteca Nazionale

Ms. C. 117, f. 153V (secolo XV), Cetus, Orion; Ibid., tav. LXXI, fig. 180, Kew, Sir Sidney C. Cockerell, De ymaginibus celi, f. 3r (secolo XV), Andromeda; Ibid., tav. LXXII, tav. 183, Cambridge, Emmannuel College, Ms. 70, f. 104r (secolo XV), Cetus.

90 Stuttgart Württemberische Landesbibliothek, Cod. bibl. fol. 23, fol. 79 http://warburg.sas.ac.uk/vpc/VPC_search/record.php?record=32035.

${ }^{91}$ Stuttgart Württemberische Landesbibliothek, Cod. bibl. fol. 23, fol. 147V, http://warburg.sas.ac.uk/vpc/VPC_search/record.php?record=32230.

${ }_{92}^{2}$ HRABANUS MAURUS, De rerum naturis, codice Casin. 132. Archivio dell'abbazia di Montecassino, riproduzione fac-simile del codice 132, a cura di G. CAVALLO, Torino, 1994, p. 211 A - 213 B.

${ }_{93}$ A. RUMPF, op. cit. (n. 44), passim; M. BOOSEN, op. cit. (n. 45), passim; P. ZANKER, B. C. EWALD, op. cit. (n. 47), passim.

${ }_{94}$ A. PERONI, Il monastero altomedievale di S. Maria «Teodote» a Pavia. Ricerche urbanistiche e architettoniche, Studi medievali, ser. 3, a. XIII, 1, 1972, p. 1-93, 89-93, tavv. XXX; G. FORZATTI GOLIA, S. Maria «Teodote» di Pavia, in Monasteri in Lombardia, a cura di G. PICASSO, Milano, 1980, p. 223-235.

${ }^{95}$ La letteratura critica ha individuato le influenze dell'arte orientale, in particolare di quella sasanide, sulle decorazioni scultoree di produzione "longobarda", ma sarebbe opportuno indagare ulteriormente in questa direzione per verificare l'ipotesi, cfr. G. PANAZZA, Lapidi e sculture paleocristiane e preromaniche in Pavia, in Arte del primo millennio, Torino, 1953, p. 211-292: 215; G. DE FRANCOVICH, Osservazioni sull'altare di Ratchis e Cividale e sui rapporti tra Occidente ed Oriente nei secoli VII e VIII d.C., in Scritti di Storia dell'arte in onore di M. Salmi, I, Roma, 1961, p. 173-236; C. GABERSCEK, L'eredità sassanide in Friuli, in Memorie storiche forogiuliesi, 51, 1971, p. 18-37: 23-24. In questo caso ci sembra che, proprio per il retaggio classico dei mostri alati con funzione di psicopompi, si potrebbe anche riprendere l'ipotesi di una funzione funeraria della lastra.

${ }^{96}$ La ricerca, ancora parziale, è stata realizzata sul sito della Biblioteca Nazionale di Parigi, con i motori di ricerca Mandragore e Gallica, per i manoscritti in questione, cfr. Paris, Bibliothèque Nationale de France (=BNF), Gregorius Nazianzenus, Orationes, Grec. 510, fol. 3, Giona rigettato dal mostro (secolo IX). <http://visualiseur.bnf.fr/Visualiseur?Destination=Mandragore\&O=08452208\&E=19\&I=112078\&M=imageseule>; Paris, BNF, Johannes Damascenus, Sacra parallela, Grec 923, fol. 29v, Giona gettato in mare (seconda metà secolo IX) <http://visualiseur.bnf.fr/Visualiseur?Destination=Mandragore\&O=0 7907204\&E=1\&I=151599\&M=imageseule>.

${ }_{97}$ Paris, BNF, Cantica, Supplément grec 1335, fol. 331v (manoscritto di provenienza cipriota, seconda metà secolo XII) <http://visualiseur.bnf.fr/Visualise ur? Destination=Mandragore $\& \mathrm{O}=08100985 \& \mathrm{E}=46 \& \mathrm{I}=94753 \& \mathrm{M}=$ imageseule $>$.

${ }_{98}^{8}$ Paris, BNF, Bible de Roda, lat. 6 (3), f. 83r, Giona inghiottito e rigettato dalla balena (metà- terzo quarto XI secolo) <http://visualiseur.bnf.fr/Visualiseu $r$ ?Destination=Mandragore $\& \mathrm{O}=07920323 \& \mathrm{E}=1 \& \mathrm{I}=53619 \& \mathrm{M}=$ imageseule $>$; cr. F. POMARICI, op. cit. (n. 57), p. 64.

99 Paris, BNF, Bibbia, Lat. 16528, fol. 311, Giona inghiottito dalla balena (manoscritto di provenienza italiana datato al 1188) <http://visualiseur.bnf.fr/ CadresFenetre?O=COMP-11\&M=imageseule>.

${ }^{100}$ Un pesce compare anche nell'immagine dedicata a Giona dell'Hortus deliciarum di HERRADE DE LANDSBERG (1167-1185), cfr. F. POMARICI, op. cit. (n. 57), p. 64, fig. 3 


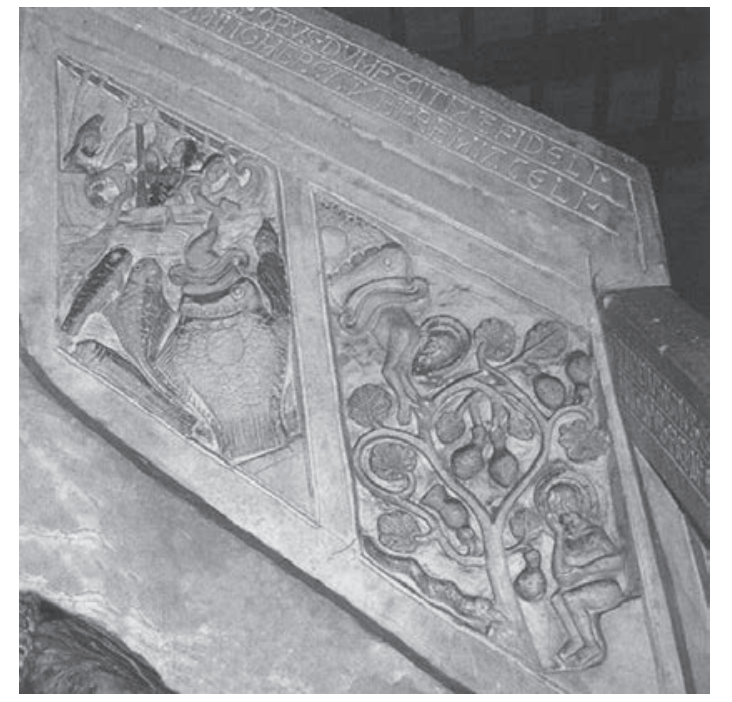

Fig. 15. Moscufo, S. Maria del Lago, Ambone, Storia di Giona, 1159 (da N. ZCHOMELIDSE, Art, Ritual and Civic Identity in Medieval southern Italy, University Park, PA, 2014, fig. 170)

di Parigi ${ }^{100}$. Nei manoscritti, inoltre, l'immagine del kētos viene usata anche come motivo decorativo, privo di uno specifico significato, per decorare, ad esempio, i capilettera delle Bibbie Atlantiche, sia nelle forme classiche ${ }^{101}$ che in quelle alate ${ }^{102}$.

(fig. 15) Nel secolo XII, il mostro che inghiotte Giona è chiaramente raffigurato come un grande pesce anche in scultura, per esempio sugli amboni eseguiti da Roberto e Nicodemo nelle chiese di S. Maria in Valle Porclaneta a Rosciolo (1157) e di S. Maria del Lago a Moscufo (1159) ${ }^{103}$.

La presenza della storia di Giona sugli amboni delle chiese serviva, da un punto di vista didattico, a valorizzare il simbolismo cristologico, di morte e resurrezione, con il quale l'episodio veniva interpretato, in base al Vangelo di Matteo $^{104}$. In particolare, in Italia meridionale, la raffigurazione del racconto biblico sugli amboni aveva anche una funzione liturgica durante lo svolgimento della cerimonia del Sabato santo, che prevedeva la lettura del libro di Giona e la recitazione del preconium pasquale riportato sui rotoli di exultet, culminando con l'accensione del cero pasquale, eseguita proprio dall'ambone ${ }^{105}$. Non a caso, infatti, proprio le illustrazioni dei rotoli di exultet ci consegnano una significativa testimonianza della raffigurazione della storia

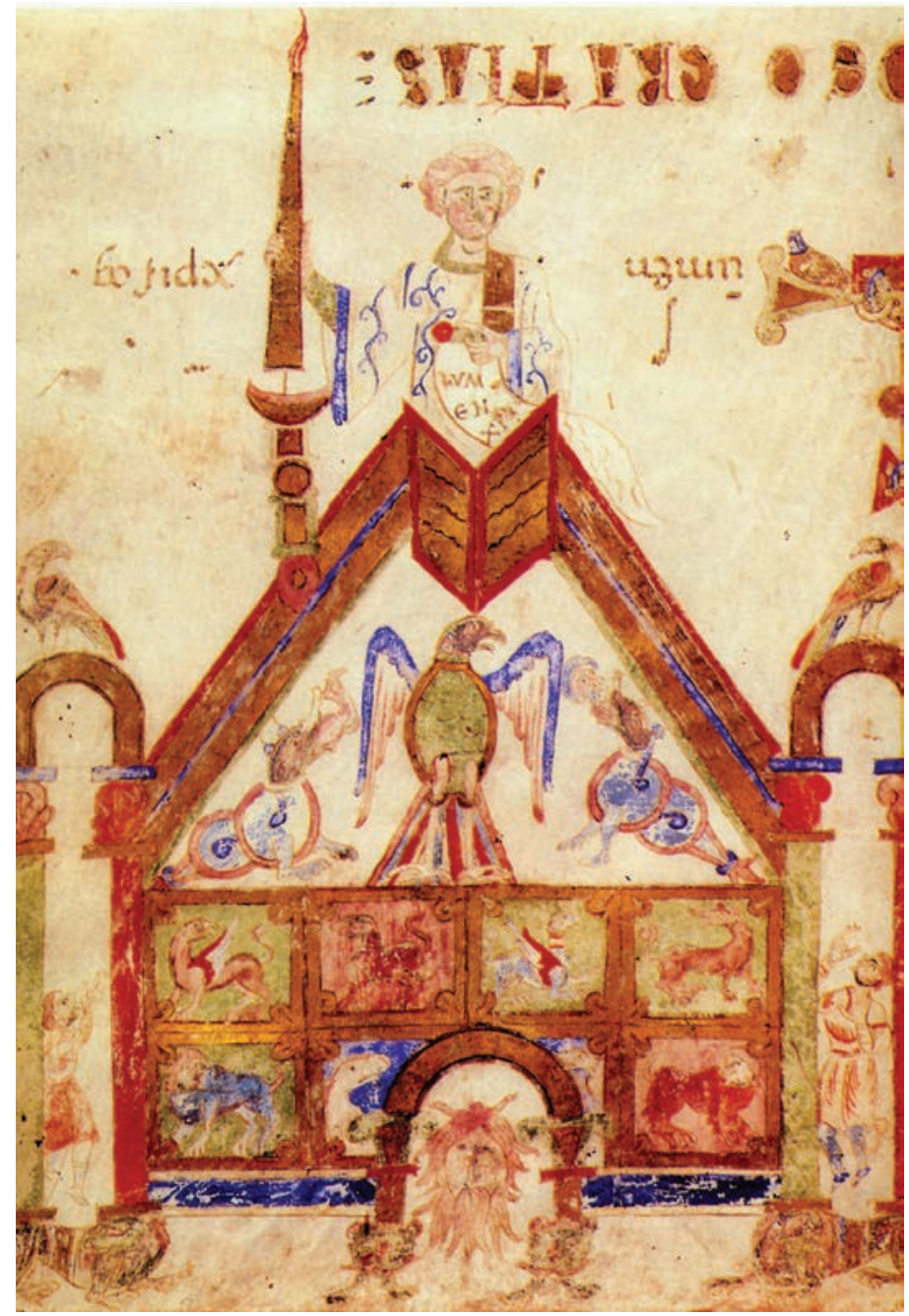

Fig. 16. Pisa, Museo dell'Opera del Duomo, Exultet 2, Accensione del cero, secolo XII (da Exultet. Rotoli liturgici del medioevo meridionale, a cura di G. CAVALLO, Roma 1994)

di Giona, e del kētos, nelle scene che ritraggono l'ambone con il chierico intento nella celebrazione ${ }^{106}$. La creatura ritratta sugli amboni segue il modello antico della creatura marina dalla doppia natura (cane-leone e pesce-serpente), ma non del kétos classico. (fig. 16) Nell'Exultet di Pisa, eseguito forse a Capua, in ambito cassinese, tra il 1059 e il $1071^{107}$, oppure nell'Exultet di Mirabella Eclano (secolo

\footnotetext{
${ }^{101}$ Paris, BNF, Par. lat. 104, f. 168r, Iniziale della Lettera ai Colossesi (secolo XII), cfr. E. COTTEREAU, scheda, in Le Bibbie Atlantiche. Il libro delle scrittura tra monumentalità e rappresentazione, a cura di M. MANIACI, G. OROFINO, Milano, 200o, p. 155-158: 157, fig. 12.

${ }^{102}$ Napoli, Biblioteca Nazionale Vittorio Emanuele III, Neapol. VI B 7, f. 133r (secolo XII), cfr. S. SCIPIONI, scheda, in Le Bibbie Atlantiche, p. 213-217: 214, fig. 28; Città del Vaticano, Biblioteca Apostolica Vaticana, Vat. Ross. 617, f. 94r, iniziale di Esther (secolo XII med.), cfr. M. SIGNORINI, scheda, in Le Bibbie Atlantiche, p. 191-193: 192, fig. 19.

${ }^{103}$ L. AVENTIN, Des images au service de la parole. Le programme iconographique des ambons de Rosciolo, Moscufo e Cugnoli (Abruzzes, 1157-1166), in Cahiers de civilisation médiévale, 46, 2003, p. 301-326; F. GANDOLFO, Scultura medievale in Abruzzo. L'età normanno sveva, Pescara, 2004, p. 69-75 (Rosciolo), 76-83 (Moscufo).

${ }^{104}$ Mt. 12, 40.

${ }^{105}$ A. A. KING, Liturgie of the Past, Rites of Western Christendom, IV, London, 1959, p. 52-76; Dorothy Glass ha collegato la raffigurazione del libro di Giona alla Lectio Jonae prophetae cum cantico durante il Venerdì santo (in alcuni casi anche durante il Sabato santo), secondo l'uso attestato nei manoscritti beneventani, cfr. D. GLASS, Jonah in Campania. A Late Antique revival, in Commentari. Rivista di critica e storia dell'arte, XXVII, 3-4, 1976, p. 179-193: 184185. Sulla liturgia della laus cerei connessa ai rotoli di exultet, R. RONZANI, Il mito delle fonti della laus cerei e il testo dell'Italia meridionale longobarda, in Hagiologia. Studi per Réginald Grégoire, 2 voll., a cura di A. BARTOLOMEI ROMAGNOLI, U. PAOLI, P. PIATTI, Fabriano, 2012, II, p. 1123-1142; N. ZCHOMELIDSE, Art, Ritual and Civic Identity in Medieval southern Italy, University Park, PA, 2014, p. 34-72.

${ }^{106}$ D. GLASS, op. cit. (n. 97), p. 179-183; N. ZCHOMELIDSE, op. cit. (n. 97), p. 73-169. Sui rotoli di exultet si vedano le singole schede in Exultet. Rotoli liturgici del medioevo meridionale, a cura di G. CAVALLO, Roma, 1994.

${ }^{107}$ A. R. CALDERONI MASETTI, L'exultet "beneventano" del Duomo di Pisa, Galatina, 1989, p. 46-48; EAD., Pisa Museo dell'Opera del Duomo, Exultet 2, in Exultet op. cit. (n. 98), p. 151-157.
} 


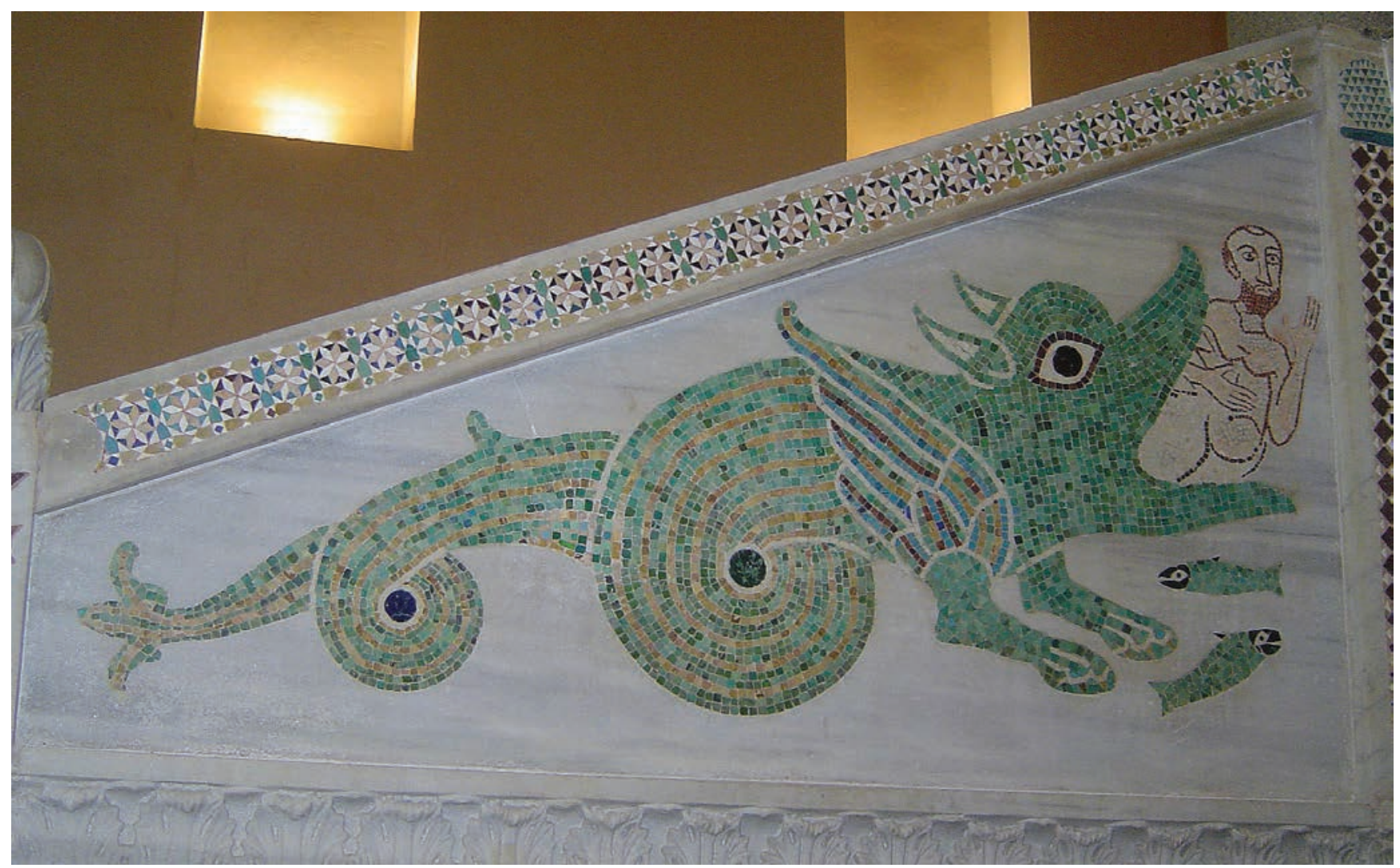

Fig. 17. Ravello, Cattedrale, Ambone, Giona inghiottito dal kētos, 1094-1150

$\mathrm{XI})^{108}$ che reca solo il mostro marino, senza Giona, la creatura non ha il muso allungato, il collo è corto e le orecchie sono poco pronunciate, suggerendo che il modello fosse l'animale presente nel thiasos marino dei sarcofagi antichi, ritratto con testa da canide. Da notare che in un solo caso la creatura è raffigurata con le ali, nell'Exultet del Tesoro della Cattedrale di Capua ${ }^{109}$.

Partendo quindi dal modello classico di un mostro marino con la testa canina, ma non più del kētos classico, gli scultori medievali dell'Italia meridionale realizzarono la decorazione della lastra sulla porta del campanile di S. Maria Assunta a Positano (secolo XI), mentre il pluteo del Museo Correale di Terranova (Sorrento) prende a modello un leone marino, con una bella criniera in evidenza (prima metà secolo XII)

(fig. 17) Anche gli amboni che riportano la storia di Giona, disseminati nell'area campana e nel Basso Laziono, come quello di Ravello che è l'unico conservato intatto, non ritraggono più il kētos classico ma una creatura alata.
In questo caso abbiamo una datazione certa al tempo del vescovo Costantino Rogadeo tra il 1094 e il $1150^{\mathrm{mt}}$.

Una simile iconografia si riscontra anche in un frammento di pulpito ora nel Museo Provinciale Campano a Capua (fine XI secolo). Si tratta di un pannello triangolare, ne resta una metà, che rappresenta la parte superiore del corpo di un mostro marino alato che lascia uscire Giona dalle fauci" ${ }^{12}$. (fig. 18) Anche a S. Pietro a Minturno, in un pluteo reimpiegato nel pulpito (secolo XII), si trova un mostro marino alato che inghiotte Giona ${ }^{\text {113 }}$. Esempi in tal senso proseguono fino alla prima metà del Duecento, ma mostrano un progressivo abbandono delle forme della creatura marina di derivazione classica (Ravello, S. Giovanni del Toro ${ }^{14}$; Gaeta, cattedrale, lastre murate sul campanile ${ }^{\mathrm{nI}}$ ), a vantaggio di un animale che è a tutti gli effetti ritratto come un pesce alato (Cattedrale di Alatri, lastra di Giona $\left.{ }^{16}\right)$, (fig. 19) per giungere ad un semplice "grande pesce", privo di ali (Sessa Aurunca, lastre di pluteo, datate $\left.1224-1259^{117}\right)$.

\footnotetext{
${ }^{108}$ G. OROFINO, scheda, in Exultet op. cit. (n. 97), p. 303-318.
}

${ }^{109}$ M. AVERY, The Exultet Rolls of South Italy, II, Plates, Princeton, 1936, tav. XXIX.

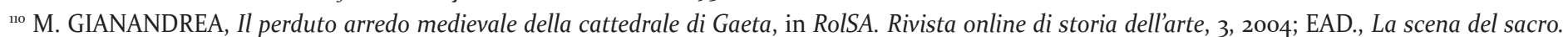
L'Arredo liturgico nel Basso Lazio tra XI e XIV secolo, Roma, 2006, p. 37-38.

${ }^{m}$ P. PENSABENE, Il reimpiego a Ravello. Il caso del duomo, in Rivista dellistituto nazionale d'Archeologia e Storia dell'arte, ser. III, XXXIV, 66, 2011 [ma 2015], p. 57-95: 89-91.

${ }^{12}$ M. GIANANDREA, La scena del sacro op. cit. (n. 102), p. 300, fig. 231; E. SCIROCCO, op. cit. (n. 6o), p. 99, fig. 6.

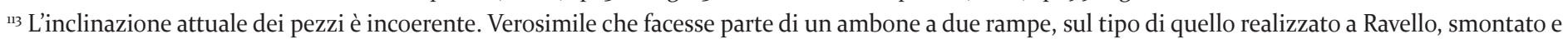
rimontato in questo modo nel 1618, cfr. M. GIANANDREA, La scena del sacro op. cit. (n. 102), p. 159-164, 273, fig. 140-141.

${ }^{114}$ L'ambone della chiesa di S. Giovanni del toro a Ravello, Ravello, 1991; F. GANDOLFO, op. cit. (n. 95), p. 96-10o.

${ }^{115}$ M. GIANANDREA, La scena del sacro op. cit. (n. 102), p. 97-102, 103-106, 234, fig. 32-33.

${ }^{116}$ Ibid., p. 90-92, 232 fig. 27.

${ }^{17}$ L. COCHETTI, I rilievi della cattedrale di Sessa Aurunca e lo sviluppo dei marmorarii "neocampani" nel XIII secolo, in Commentari, 9, 1958, p. 75-87; V. Pace, Il programma decorativo nel XIII secolo, in M. D’ONOFRIO, V. PACE, A. M. VILLUCCI, La cattedrale di Sessa Aurunca, Sessa Aurunca, 1983, p. 29-39; F. GANDOLFO, La scultura normanno-sveva in Campania: botteghe e modelli, Roma, 1999, p. 111-115. 


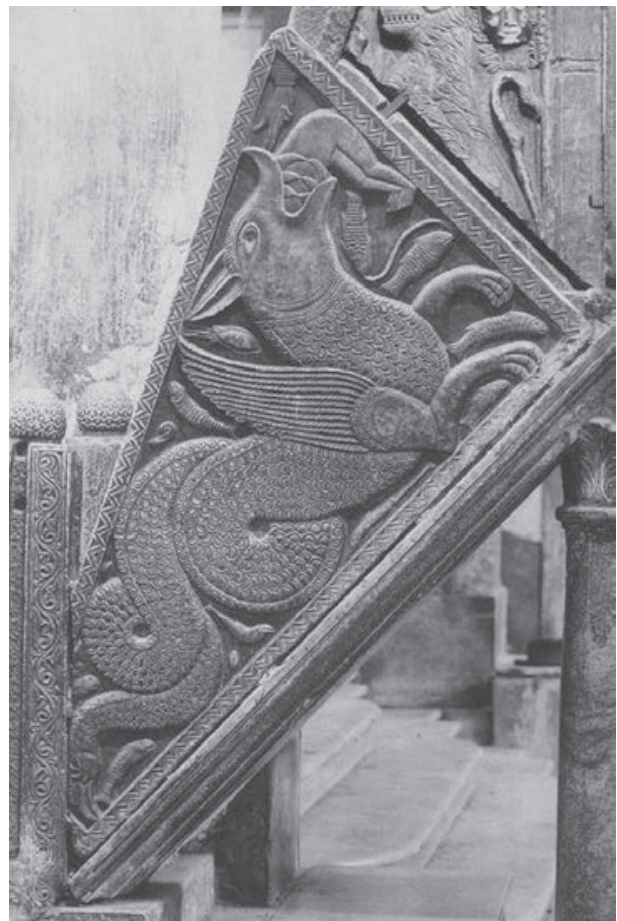

Fig. 18. Minturno, Collegiata S. Pietro, Lastra di ambone, Giona inghiottito dal kētos, fine secolo XI (da N. ZCHOMELIDSE, Art, Ritual and Civic Identity in Medieval southern Italy, University Park, PA, 2014, fig. 49)

\section{IL KETTOS E IL SENMURV}

Una suggestiva ipotesi sulla riformulazione iconografica del motivo del kètos, dettata anche dall'osservazione della presenza delle ali sul corpo della "balena", venne proposta da Wolfgang Volbach, secondo il quale la trasformazione del mostro marino deriverebbe dalla sua contaminazione con il sēnmurv sasanide, riprodotto nei tessuti bizantini, e da qui reinterpretato dagli artisti campani, attorno al secolo $\mathrm{XI}^{118}$. Questa ipotesi ha incontrato attenta considerazione condizionando gli studi successivi. In particolare, Giovanni Curatola ha cercato una soluzione nell'ambito della traduzione armena della Bibbia, confluita poi nella decorazione della chiesa di S. Croce a Aght'Amar, individuando una sorta di sdoppiamento semantico del kētos, presente nella traduzione in armeno della Bibbia, raffigurato come un pesce quando inghiotte Giona e come un vishap (ovvero un sēnmurv), cioè alato, allorquando beneficamente rigetta il profeta, restituendogli la vita ${ }^{119}$. La comune fonte bizantina responsabile
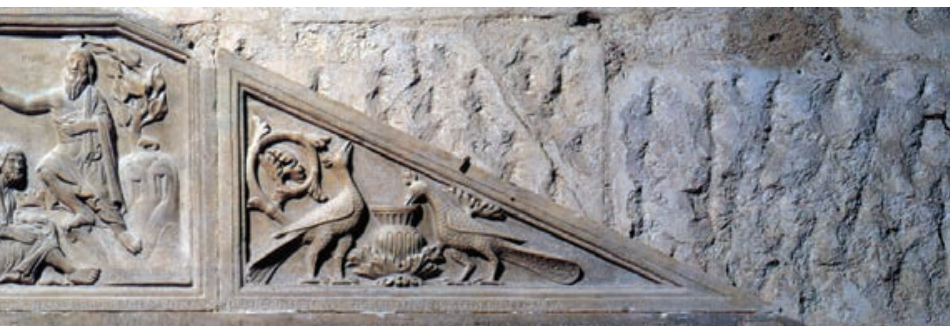

.
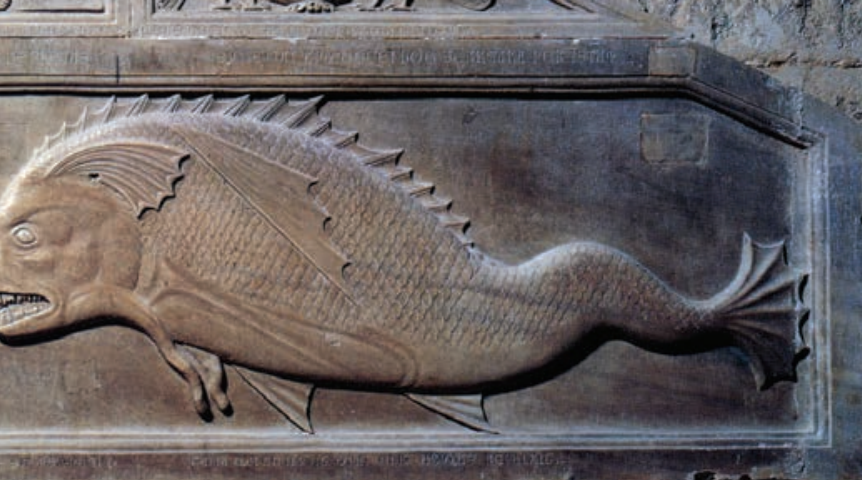

Fig. 19. Sessa Aurunca, Cattedrale, Lastra di ambone, Magister Peregrinus, Giona rigettato dal kētos, 1259-1283

della trasmissione del sēnmurv e della conseguente trasformazione iconografica del kētos, sia per l'Armenia che per la Campania, è stata presa in seria considerazione anche da Manuela Gianandrea ${ }^{120}$ e da chi scrive ${ }^{121}$. Più recentemente anche Francesca Pomarici è tornata sull'argomento, con osservazioni critiche, ma sempre partendo dall'assunto che al kētos vennero aggiunte le ali ${ }^{122}$. Sull'argomento si è pronunciata, infine, Elisabetta Scirocco, dimostrandosi poco incline ad accogliere l'ipotesi di Volbach ${ }^{123}$.

Per quanto riguarda la penisola italiana, l'impostazione del problema, cioè pensare alla riformulazione iconografica di un kētos al quale spuntano le ali, appare del tutto fuorviante. Come abbiamo visto, infatti, nell'arte antica (greca, etrusca e romana), alcune creature marine sono raffigurate con le ali, e il kētos stesso presenta una variante alata, soprattutto quando raffigurato da solo in veste di psicopompo nei monumenti funerari. Inoltre, il kētos "alato" entra anche nell'iconografia di Giona ${ }^{124}$, mentre creature marine alate erano già apparse nell'ambito dell'arte longobarda, sulla lastra di Teodote. D'altro canto, il ricorso generico alla circolazione dei tessuti orientali in Occidente per giustificare la presenza di temi sasanidi nell'arte occidentale, è una teoria che si è dimostrata priva di concreto fondamento, se non circostanziata caso per caso ${ }^{125}$.

Per quanto riguarda il tema del sēnmurv, occorre qui fare una precisazione. Secondo gli studi recenti di Matteo Com-

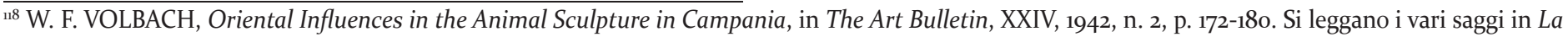
seta e la sua via, cat. mostra (Roma 1994), a cura di M. T. LUCIDI, Roma 1994.

"19 Giovanni Curatola chiama la creatura Vishap-Arlez, cfr. G. CURATOLA, Il «Vishap» di Aght'Amar: nota sulla diffusione occidentale di un motivo iconografico, in Oriente moderno, LVIII, 7-8, 1978, p. 285-302: 285-286, 290, 301 nota 3. Anche Ajello ha fornito un accurato studio sul motivo del cane alato, cfr. R. AJELLO, Sulle divinità armene chiamate "Arlez", in Oriente Moderno, LVIII, 7-8, 1978, p. 303-316.

${ }^{120}$ M. GIANANDREA, Il perduto arredo op. cit. (n. 102); EAD., La scena del sacro op. cit. (n. 102), p. 37-39.

${ }^{122}$ S. RICCIONI, La rappresentazione della natura tra ornamento e narrazione. Il bestiario di Roma tra i secoli XI e XII, in Medioevo: natura e figura. La raffigurazione dell'uomo e della natura nell'arte medievale, Atti del convegno internazionale di studi dell'AISAME (Parma 20-25, sett. 2011), a cura di A. C. Quintavalle, Milano, 2015, p. 342-343.

${ }^{122}$ F. POMARICI, op. cit. (n. 57), p. 64.

${ }^{123}$ E. SCIROCCO, op. cit. (n. 60), p. 99-100.

${ }^{124}$ In un manoscritto greco che raffigura Giona rigettato dal mostro, il kētos ha coda di serpente attorcigliata e pinne che salgono in verticale come vere e proprie ali, cfr. Paris, Bibliothèque Nationale, Cantica, Supplément grec 1335, fol. 331v, seconda metà secolo XII.

${ }^{125}$ J. LECLERCQ-MARS, L'imitation des tissus "orientaux" dans l'art du haut moyen age et de l'époque romane, in Medioevo mediterraneo. L'Occidente, Bisanzio e l'Islam. Atti del convegno internazionale di studi dell'AISAME (Parma, 21-25, sett. 2004), a cura di A. C. QUINTAVALLE, Milano, 2007 , p. 456-469: 456.
} 


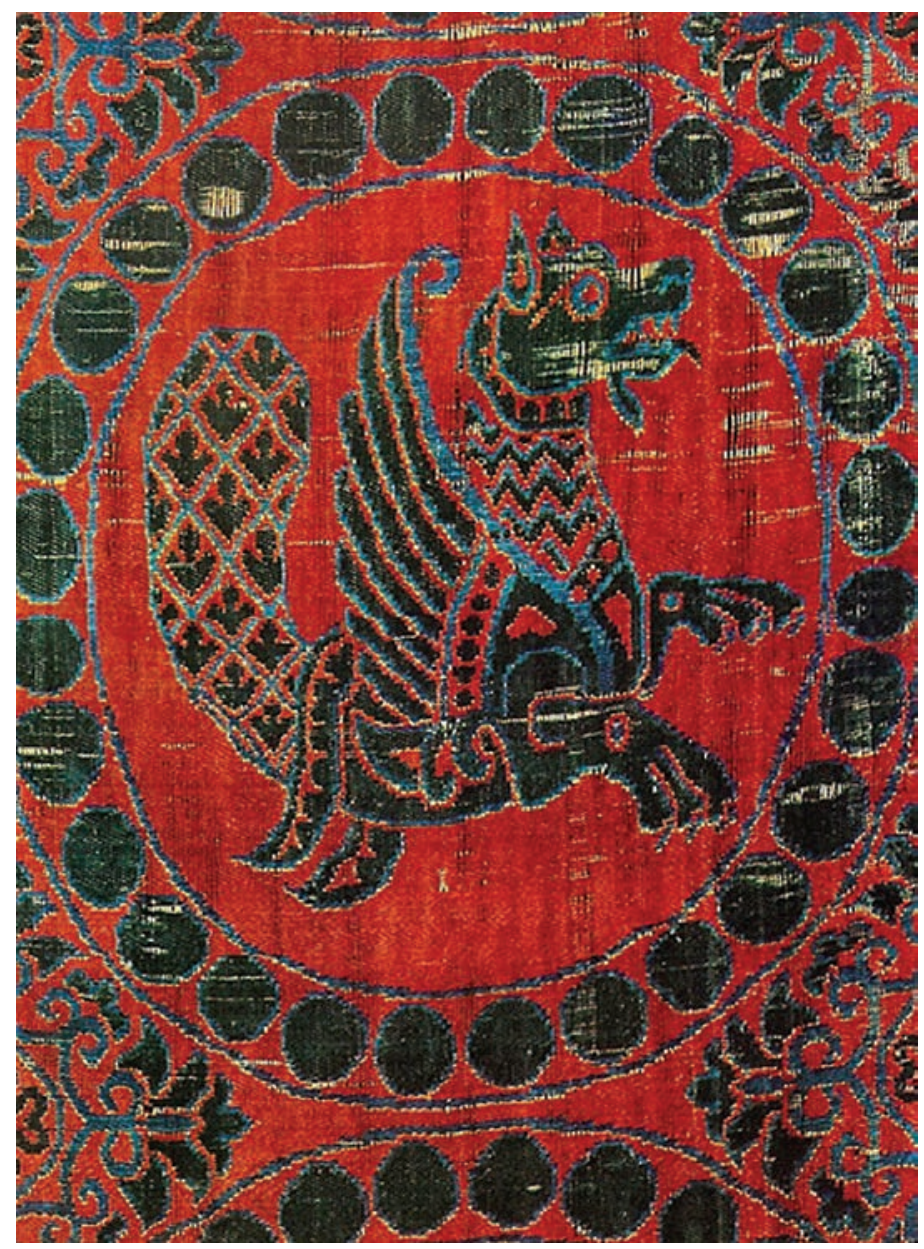

Fig. 20. Firenze, Museo Nazionale del Bargello, tessuto in seta, Sēnmurv, secolo VI-VII (da R. GHIRSHMAN, Arte persiana. Parti e Sassanidi, Milano, 1962, p. 229, fig. 276)

pareti, il vero sēnmurv che si trova raffigurato nei manoscritti persiani sarebbe un uccello, molto simile alla fenice cinese, giunto in Persia al tempo della dominazione mongola. (fig. 2o) Quindi l'animale alato, con testa di canide, zampe di felino e coda di pavone (talvolta di pesce), diffuso nell'arte sasanide e nella prima arte islamica, sarebbe uno pseudosēnmurv e andrebbe interpretato come Gloria divina, in quanto espressione della divinità Mazda (versione iraniana dello Zoroastrismo), e simbolo protettore della famiglia regnante dei Sasanidi (224-640) ${ }^{126}$. Questo pseudo-sēnmurv, si trova raffigurato sui bassorilievi rupestri di Taq-i Bustān, in Iran, non lontano da Behistun, sulla via della seta. In particolare la creatura è raffigurata con coda di pavone, sulle vesti del re intento nella caccia al cinghiale. Essa si trova nei piatti d'argento parthici e sasanidi, sui tessuti e i tappeti, sulle decorazioni in stucco, sui vasi metallici dall'alto collo che giungeranno fino al periodo islamico. Da qui passerà nella cultura bizantina e verrà introdotto in Occidente.

Esisteva però un animale, per alcuni assimilabile al sēnmurv, molto simile alle creature marine dell'arte greca e romana, che nelle pitture sogdiane di Panjakant - nell'odierno Tagikistan - (metà secolo VIII ca.) è raffigurato con testa di pantera/canide, coda che somiglia a quella di un serpente o di un pesce e li $^{127}$. Questo animale, secondo Compareti, sarebbe frutto del reimpiego da parte dei persiani di quei mostri acquatici, esseri ctoni per eccellenza, spesso cavalcati dalle Nereidi o da altre simili divinità che abbiamo considerato in apertura del lavoro e visibili sui monumenti funerari dei sogdiani sepolti a Xi'an nel VI secolo ${ }^{128}$.

L'ipotesi di un influsso che da Oriente si sarebbe mosso verso Occidente, appare quindi completamente ribaltata in direzione opposta. Furono probabilmente i Greci che, per effetto della conquista macedone sui persiani, esportarono queste iconografie nell'antica Persia, condizionando la resa di alcuni motivi decorativi e in particolare quello del sēnmurv.

Alla luce di quanto qui evidenziato per quanto riguarda la tradizione iconografica del kètos in Occidente, ci sembra quindi che non vi sia più alcuna necessità di ipotizzare una contaminazione con l'arte persiana, e in particolare con l'ippocampo o il sēnmurv per giustificare la presenza delle ali negli animali che figurano negli amboni campani (e non solo). Sembra più plausibile, invece, ritenere che a partire dell'età paleocristiana, quando il kētos "classico", spesso nella versione alata, venne adottato per rappresentare il piscem grandem della Vulgata, le sue forme abbiano subito una mutazione avvicinandolo alla pantera/cane del corteo marino, anche per effetto del suo progressivo scollamento dalla tradizione ebraica che indicava l'animale biblico come tanin (cioè un coccodrillo-serpente). Gli scultori campani dovevano avere numerosi esempi di opere romane dalle quali attingere i modelli per questa creatura ${ }^{129} \mathrm{e}$, inoltre, negli anni in cui lavoravano, il recupero di temi provenienti dall'antichità era una pratica diffusa e, talvolta, connotata da significati ideologici ${ }^{130}$.

Furono quindi questi generici kètēe, in particolare la pantera marina alata, a sostituire il kētos "classico" nella raffigurazione della vicenda di Giona nell'Italia meridionale. Proprio la presenza di questi animali sui sarcofagi, quindi il loro significato funerario che evocava la vicenda di morte e rinascita di Giona, oltre alla perdita del contatto con il significato che il kètos aveva durante l'età paleocristiana,

\footnotetext{
${ }_{126}$ A. M. BELENITSKII, B. I. MARSHAK, The Paintings of Sogdiana, in G. AZARPAY, Sogdian Painting, Berkeley - Los Angeles, 1981, p. 11-77: 70, 73; B. I. MARSHAK, Legends, Tales, and Fables in the Art of Sogdiana, New York, 2002, p. 37; ID., Zoroastrian Art in Iran under the Parthians and the Sasanians, in PH. J. GODREJ, F. P. MISTREE (eds.), A Zoroastrian Tapestry. Art, Religion \& Culture, Usmanpure, 2002, p. 134-147: 140-141; M. COMPARETI, The So-called Sēnmurv in Iranian art: A Reconsideration of an Old Theory, in Loquentes linguis. Studi linguistici e orientali in onore di Fabrizio A. Pennacchietti, a cura di P. G. BORBONE, A. MENGOZZI, M. TOSCO, Wiesbaden, 2006, p. 185-200; ID., S. CRISTOFORETTI, Il fumo iranico della pipa di Perm, in Multa e Varia. Studi offerti a Maria Marcella Ferraccioli e Gianfranco Giraudo, a cura di F. CRET CIURE, V. NOSILIA, A. PAVAN, Milano, 2012, p. $239-250$.

${ }^{127}$ B. I. MARSHAK, V. I. RASPOPOVA, A Hunting Scene from Penjikent, in Bulletin of the Asia Institute, 4, 1990, p. 77-94; B. I. MARSHAK, Legends op. cit. (n. 125).

${ }^{128}$ Ibid., p. 239-250; M. COMPARETI, Due tessuti cetrasiatici cosiddetti “zandanij” decorati con pseudo-Simurgh, in Le spigolature dell'Onagro. Miscellanea composta per Gianroberto Scarcia in occasione dei suoi ottant'anni, a cura di M. COMPARETI, R. FAVARO, Venezia, 2013, p. 17-38: 21, 25-26.

${ }^{129}$ Questa ipotesi era già stata formulata da D. GLASS, op. cit. (n. 97), p. 185-186.

${ }^{130} \mathrm{Al}$ riguardo la bibliografia è assai vasta, si veda almeno H. TOUBERT, Un art dirigé. Réforme grégorienne et iconographie, Paris, 1990. Per una rassegna del dibattito critico si veda S. RICCIONI, La décoration monumentale à Rome aux XI et XII e siècles: révisions chronologiques, stylistiques et thématiques, in Perspective, 2, 2010-2011, p. 319-360: 321-324.
} 
può aver indotto committenti e artisti ad adottare questi modelli. Motivi di questo kētos alato, ne troviamo disseminati in gran parte della produzione artistica della penisola, in Molise a S. Giorgio Martire a Petrella Tifernina ${ }^{131}$, associati alla storia di Giona; in Abruzzo, nella iconostasi di S. Maria in Valle Porclaneta (1140-1150) ${ }^{132}$, nel portale di S. Giovanni ad insulam (inizio secolo XIII) ${ }^{133}$, nei più tardi plutei di S. Pellegrino a Bominaco ${ }^{134}$, e della cattedrale di S. Massimo $(1263)^{135}$; in Veneto, nel frammento di mosaico della cripta della cattedrale di Treviso (secolo XII) ${ }^{136}$.

Infine, in alcuni specifici casi, ma per altre vie, la contaminazione dalle decorazioni orientali pare ancora accettabile, a causa delle caratteristiche morfologiche dell'apparato decorativo che mostra la ricezione di elementi propri dell'Oriente islamico, conosciuti verosimilmente attraverso le stoffe di lusso provenienti dalle manifatture asiatiche, bizantine, e mediate dall'interpretazione dell'area arabo-siciliana ${ }^{137}$. Come nel caso dei plutei del Museo Campano di Capua e della cattedrale di Minturno, dove il "kētos" reca al punto dell'attacco dell'ala al corpo una sorta di anello perlinato, che appare come una derivazione dalla produzione tessile sassanide, presente anche nella scultura pugliese e sarda ${ }^{138}$.

Per concludere, in Occidente, l'immagine del kētos "classico", spesso nella versione alata, che fin dalla tarda antichità venne adottata per raffigurare il grande pesce che inghiotte Giona, sui sarcofagi e nelle catacombe, durante l'alto medioevo si confonde con quella del cane/pantera marino, anche alato come nella particolare produzione campana, fino a scomparire almeno a partire dal secolo XII. Nella storia di Giona si fa largo progressivamente una trasposizione figurativa che segue la lettera del piscem grandem della Vulgata latina. Esemplare in tal senso, nel secoloXIII, la raffigurazione della scena nella cattedrale di Gaeta, sempre nell'Italia del Sud, dove il kètos mega è ormai solo un pesce molto grande. Questa creatura troverà poi larga diffusione anche come motivo decorativo isolato, conservando però sempre un significato simbolico negativo, fino a confondersi con il drago.

\footnotetext{
${ }_{131}$ Nella lunetta del portale principale è ritratta la storia di Giona, inghiottito e rigettato dal kētos, una creatura simile è ritratta anche a S. Maria della Strada presso Matrice, cfr. F. POMARICI, L'agnello e il liber creaturarum: il programma iconografico delle lunette, in Il cantiere della chiesa di San Giorgio Martire a Petrella Tifernina, a cura di M. GIANANDREA, W. ANGELELLI, F. GANDOLFO, F. POMARICI, Roma, 2012, p. 87-103: 87-91, e nota 8.

${ }^{132}$ Pluteo destro dell'iconostasi, F. GANDOLFO, Scultura op. cit. (n. 103), p. 57-59, fig. 107.

${ }_{133}$ F. GANDOLFO, op. cit. (n. 95), p. 171-172, fig. 275 e 280, p. 173 (Gandolfo indica l'animale come un sēnmurv).

${ }_{134}$ Pluteo di sinistra della recinzione presbiteriale, cfr. Ibid., p. 199-200, fig. 346.

135 Pluteo frammentario conservato al museo di Penne, cfr. Ibid., p. 199-200, fig. 345.

${ }^{136}$ X. BARRAL I ALTET, Les mosaïques de pavement medievales de Venise, Murano, Torcello, Paris, 1985, p. 93, 191 fig. 213.

${ }^{137}$ Sull'arte islamica in Italia, cfr. U. SCERRATO, Arte islamica in Italia, in Gli arabi in Italia. Cultura, contatti e tradizioni, a cura di F. GABRIELI, U. SCERRATO, Milano, 1979, p. 271-570.

${ }^{138}$ Renata Serra individuava in questo motivo un tratto peculiare della scultura sarda, da qui passato in Puglia nel secolo XI, cfr. R. SERRA, I plutei tardo bizantini dell'isola di San Macario e di Maracalagonis (Cagliari), in Archivio Storico Sardo, 30, 1976, p. 59-76. Roberto Coroneo ha precisato l'origine del motivo in opere sasanidi, cfr. R. CORONEO, Scultura medio bizantina in Sardegna, Nuoro, 200o, p. 172, 178-194 e scheda n. 4.8, p. 220; ID., Scultura medio bizantina in Campania e in Sardegna: prototipi e modelli, in Medioevo i modelli. Atti del convegno internazionale di studi (Parma 1999), a cura di A. C. QUINTAVALLE, Milano, 2002; A. DUCCI, scheda n. 8o, in Lucca e l'Europa, un'idea di medioevo. V-XI secolo, catalogo della mostra (Lucca 2010-2011), a cura di C. BARACCHINI et al., Lucca, 2010, p. 170-171. Per analoghe considerazioni su un reliquiario romano, cfr. S. RICCIONI, Sul "bestiario" del reliquiario di san Matteo: Montecassino, Roma e la "Riforma" tra Occidente cristiano e Oriente islamico, in "Conosco un ottimo storico dell'arte. ...." Per Enrico Castelnuovo, Scritti di allievi e amici pisani a cura di M. M. DONATO, M. FERRETTI, Pisa, 2012, p. 35-42.
} 